\title{
Modular Multibody Formulation for Simulating Off-Road Tracked Vehicles
}

\author{
Mohamed A Omar ${ }^{1}$ \\ ${ }^{1}$ Mechanical Engineering Department, Taibah University, Almadinah Almonawwarah 42353, Saudi Arabia
}

Received: July 9, 2014 Accepted: July 28, 2014 Available online: August 7, 2014

doi:10.11114/set.v1i2.462

URL: http://dx.doi.org/10.11114/set.v1i2.462

\begin{abstract}
This paper presents a formulation and procedure for incorporating the multibody dynamics analysis capability of tracked vehicles in large-scale multibody system. The proposed self-contained modular approach could be interfaced to any existing multibody simulation code without need to alter the existing solver architecture. Each track is modeled as a super-component that can be treated separate from the main system. The super-component can be efficiently used in parallel processing environment to reduce the simulation time. In the super-component, each track-link is modeled as separate body with full 6 degrees of freedom (DoF). To improve the solution stability and efficiency, the joints between track links are modeled as complaint connection. The spatial algebra operator is used to express the motion quantities and develop the link's nonlinear kinematic and dynamic equations of motion. The super-component interacts with the main system through contact forces between the track links and the driving sprocket, the support rollers and the idlers using self-contained force modules. Also, the super-component models the interaction with the terrain through force module that is flexible to include different track-soil models, different terrain geometries, and different soil properties. The interaction forces are expressed in the Cartesian system, applied to the link's equation of motion and the corresponding bodies in the main system. For sake of completeness, this paper presents dynamic equations of motion of the links as well as the main system formulated using joint coordinates approach.
\end{abstract}

Keywords: tracked vehicle, multibody system, vehicle-terrain interaction, off-road vehicles.

\section{Introduction}

Tracked vehicles are used in diverse applications including: earth moving, mining and construction, agriculture, and military. Track is an essential component in tracked vehicles that transforms the rotational motion of the driving sprocket into translational motion leading to advancing the chassis of the vehicle. Tracks are designed and used to uniformly distribute the machine weight on to the ground through large contact area and generate the tractive forces necessary to move the vehicle. The ground pressure resulting from the tracked machine weight is significantly lower than that resulting from a comparable wheeled machine. This advantage allows manufacturers to design heavy machines that will not sink in the soil. Proper design of the different machine components including tracks requires accurate prediction of the loads on those components. The ability to accurately model the vehicle component is crucial capability for studying the vehicle performance using multibody simulation. Over the past two decades, multibody dynamic simulation became an integral part of product design cycle for load prediction and for optimization. Although commercial software packages (CSP) are available for multibody dynamic simulations, many of them are lacking the capability to model the complex dynamics of tracked vehicles with enough details to satisfy the designer's needs.

The main components of track, as shown in Figure 1, consists of the roller frame, driving sprocket, carrier roller, idler, link-roller, and track-chain. The track chain is composed of identical links forming closed and cyclic system. The machine configuration could be designed with oval track-chain or triangular track-chain based on the application requirements. The track links cycle in a closed path relative to the vehicle chassis. As the track links circulate through the closed path, each track-link have interacted with every driving sprocket, idler, carrier-roller, track-roller, and the terrain. One of the major challenges in modeling tracked vehicles is to accurately model the vehicle track and the contact forces. The main forces acting on the track links can be classified as: the nonlinear inertia forces of the track-link, distributed contact force between the track-links and the terrain, the contact forces between the track and drive sprockets, the contact forces between the track-links and idlers or support wheels, and the contact forces between each track link and the neighboring links. The complex geometry of track-links, the large number of contacting bodies at the same moment, the high speed impacts between sprockets and track-links poses computational challenges. 
Accounting for the distributed nonlinear inertia forces of the track is another challenge in modeling tracks using spatial multibody codes.

Some CSP have developed multibody dynamic simulation capabilities for modeling metal tracks (MSC Software, 2007). Most of these implementations simplify the contact detection algorithms by using primitive geometries for modeling the contacting bodies leading to less accurate force prediction. Other CSP utilized general purpose algorithms for modeling tack-link dynamics and the contact detection is based on full CAD geometry leading to computationally intensive simulation and time consuming analysis. Other packages implemented a simplified representation of the tracks using pre-computed parameters and pre-determined track-link path. Although the computational efficiency greatly improved, the inertial link dynamics and contact forces are not captured accurately. McCullough and Haug 1985 proposed one of the early models for tracked vehicles. The equations of motion for the vehicle, suspension system and road wheels were derived. A mechanical system super-element that represents spatial dynamics of high mobility track vehicle suspension systems was derived. The track was represented as a complex internal force element that acts between ground, wheels, and the chassis of the vehicle. Track tension was computed from a relaxed catenary.

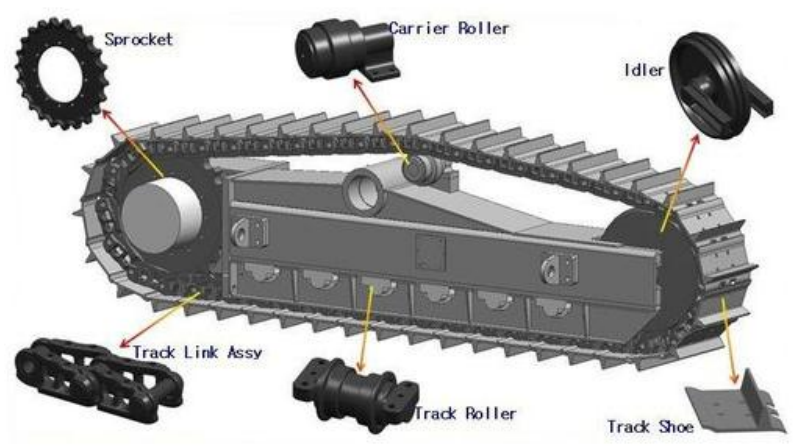

Figure 1. Major components of a typical track chain: sprocket, idler, carrier roller, track roller, track-link

(Choi, Lee, \& Shabana, 1998) proposed a spatial multibody model for a full tracked vehicle system. In this model the links were connected with revolute joints. (Choi, Lee, \& Shabana, 1998) extended their work in 1998 to develop force modules that can account for the contact between the track links and other components. The interaction between the track and terrain was modeled using simple soil mechanics based forces. (Ryu, Bae, Choi, \& Shabana, 2000) created a three-dimensional multibody model similar to that by (Ma \& Perkins 2006) and (Madsen 2007), except that the vehicle has a compliant track chain. The revolute joints which connect track links with their neighbors were replaced with compliant force elements which were described by stiffness and damping values. The model by (Ryu et al., 2000) included fairly sophisticated hydro-pneumatic suspension units. (Ryu, Huh, Bae, \& Choi, 2003) built on the methodology proposed in (Ryu et al., 2000) by further developing the contact force model to investigate the advantages of using an active track tensioner in the vehicle design. Petzold and Wehage (1994) proposed a method for formulating highly optimized vehicle models using symbolic analysis. The vehicle model makes extensive use of computation which can be pre-computed in advance of the real-time simulation.

Sandu and Freeman (2005) developed a general model of a tracked vehicle using a trailing-arm suspension system, and an independent flexible-band track model. The main purpose of the model was to study the different suspension system parameters with different number of road wheels. The developed model for tracked-vehicle was used to analyze high-speed military tracked vehicles. Ma and Perkins (2006) developed a track-wheel-terrain interaction model which can be used as a "force" super-element in a multibody dynamics code for dynamic simulation of tracked vehicles. The model by Ma and Perkins (2006) employs a nonlinear finite element representation for the track segment that is in contact with the terrain and road-wheels. The proposed model was used to simulate two different track systems, namely a continuous rubber band track and a multi-pitched metallic track, provided the finite element mesh in the track model is properly defined. The track model and solution algorithm were used to simulate a simple two-wheel system model and a full vehicle model of an M1A1 tank. Madsen 2009, demonstrated high fidelity model and simulation results of a tracked vehicle using ADAMS. (Madsen, Pechdimaljian, \& Negrut 2007) also conducted a comparative study on the track performance on different terrains and using different modeling approaches.

Modeling the track-chain requires modeling contact at three different locations: modeling the joint between the track-links, modeling the contact between the sprocket and the track-links' bushings, and modeling contact between the between the rollers and the track-rail surfaces. Two main approaches were used to model the joint between track-links: revolute joints and compliant connections. It was reported that the first approach may suffer from simulation instability due to numerical round-off errors, Choi et al 1998, and Ryu et al 2000. Although the second approach is more 
complicated, it is more robust and efficient.

To some extent the kinematics and dynamics of chain drive system is similar to the track-chain system. Reviewing the work done in modeling chain-drive is very beneficial to modeling track-chains. (Pedersen, Hansen, \& Ambrósio 2004), and Pedersen 2004 presented approaches to model the chain drive dynamics in 2D. Detailed geometric based approach for the contact detection between the sprocket and the roller was presented. An excellent continuous contact force model was proposed and implemented for the dynamic simulations. In this model, the motion was assumed to be planar and the contact occurs at single point. The connection between the links was modeled as linear-planar spring-damper element. (Pereira, Ramalho, \& Ambrósio, 2010) proposed a model for external and internal cylindrical contact and utilized it in modeling chain drives as presented in (Pereira, Ramalho, \& Ambrósio, 2010 and 2011).

Contact between the rollers and the rail surface of the track-links is complex problem because of the complex track rail geometry. Rollers could represent the carrier roller, track roller or the idler and each one has different geometry. General purpose contact detection algorithms are available and could be readily impeded in the solver Dubowsky, 1974, (Gottschalk, Lin, \& Manocha 1996), and (Flores, \& Ambrósio 2004). However due to the associated computational overheads, more efficient contact detection algorithm will be proposed in this paper. The proposed algorithm will benefit from the geometry of the rollers as surface of revolutions to optimize the contact detection process.

The performance of off-road vehicles depends on the manner in which the vehicle interacts with the terrain. As a consequence, understanding and accurate modeling of the vehicle-interaction is crucial for the design and performance analysis of tracked vehicle. (Janosi \& Hanamoto, 1961) presented his early theory for the soil stress-strain relationships. Although, the results were correlated well with the experiment, the sinkage-slip relationship needed further developments. (Bekker, 1961) proposed several empirical models for interaction between the vehicle and terrain. (Rubinstein \& Hitron, 2004) created a track-terrain interaction force model to be impeded under DADS software. The detailed dynamic description of the track, the suspension system, and the dynamic interaction between its components were modeled in the main solver. The track-link terrain interaction is modeled with a pressure-sinkage force relationship and the tractive force was calculated from shear-slip relationship. The proposed approach was used to model and simulate full vehicle dynamics. (Wong 1983), (Wong \& Preston, 1983), (Wong \& Garber, 1984) (Wong \& Bekker, 1985), (Wong 1989), and (Wong, 2001) developed several comprehensive soil modeling formulations that could be used to model track-terrain interaction. The dynamic parameters for different soils could be found experimentally.

Using implicit solvers to perform dynamic simulation of such highly nonlinear systems could be problematic and difficult to achieve because of the nature of the forces. The track-terrain forces and the contact/impact forces are hysteretic and highly nonlinear. Implicit solvers would require the derivatives of such highly nonlinear forces. Since continuous analytical derivatives may not be possible to derive, the numerical derivatives could be used. Using numerical derivative introduces artificial noise to the simulation results and the simulation becomes unstable. In order to improve the computational efficiency and reduce the simulation time, a large value for the integrator minimum time step should be used. However, using large values for the integrator minimum time step will not allow the solver to capture high speed impact leading to misleading results. On the other hand, using explicit formulation requires variable time step integrator that could efficiently and accurately track the high speed impacts between the different bodies. As a consequence, the simulation time significantly increases as the number of bodies in the system increase.

The purpose of this research is to develop an efficient, robust, portable, and modular super-element model for track-chain spatial dynamic simulation. The development focuses on the calculation efficiency by optimizing the equation structure and using preprocessing when possible. The connections between the track-links are modeled using compliant force module that allows realistic deformation and captures the necessary reaction forces. Eliminating the kinematic joints improves the computational robustness. The proposed super-element doesn't rely on specific form of multibody formulation for the main solver to maintain the generality and portability. To achieve modularity, proposed force modules are self-contained and independent of each other. This flexibility allows developer to swap any of the proposed modules with another more accurate or less expensive modules. This paper provides the details of the contact detection algorithms, the geometry description, the underlying kinematic relations, and the force relationships.

This paper is organized as follows: the following section presents the structure of tracked vehicles and geometry of the track-link box under consideration. The nonlinear kinematic and dynamic equations of motion of the multibody system are also presented. Section 3 presents kinematic and dynamic equations of motion of the track-links. Section 4 presents development of force modules used to model the interaction forces between the track-links with neighboring links, track-link with the sprockets, and track-link with the rollers. Section 5 presents the interaction between the track links and the terrain where a simplified track-soil model is presented. The algorithm for implementing the proposed model and for solving the system equation of motion is presented in Section 6. Also, consideration to include the proposed module into existing multibody code will be discussed. Example to verify the concepts will be presented in Section 7 . Concluding remarks and suggestions for future expansions will be presented in Section 8. 


\section{Kinematic and Dynamic Equations of Motion}

Tracked vehicles include: bulldozer, tracked loader, mining shovels, tracked excavators, tanks, etc. Typical structure of a tracked vehicle can be divided into the following main subsystems, as shown in Figure 2:

- The upper chassis body that consists of the driver compartment, engine, transmission, and oil and gas tanks.

- The undercarriage linkages consists of: roller frame, push-arms, cross-links, suspensions, carrier roller, track rollers, idlers, sprockets, etc..

- Track chain consists of track-links connected by pins-bushings to form a closed loop, as shown in Figure 1.

- Tooling attachments: ripper mechanism, blade mechanism, boom, and bucket mechanisms.

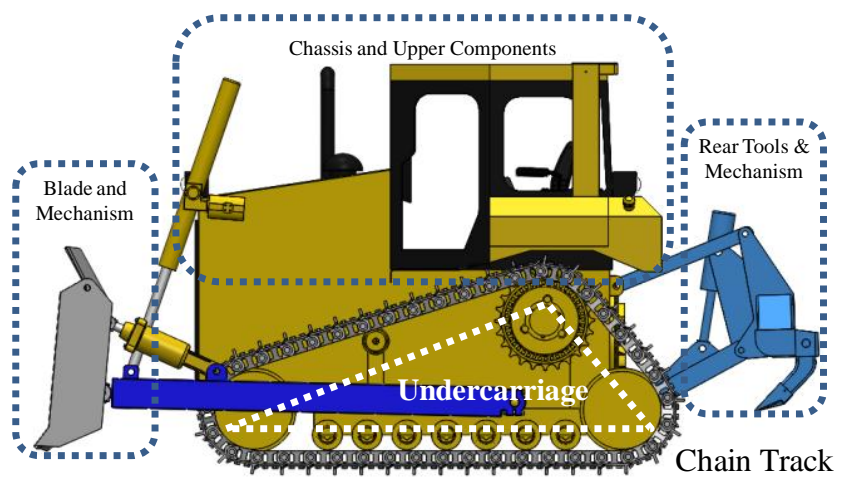

Figure 2. Major subsystems of a tacked bulldozer

This paper focuses on modeling and analysis of the track chain and its structure. The track chain is composed of identical units, as shown in Figure 1. Each unit is composed of two link-rails attached to a track shoe and a pin-bushing box, as shown in Figure 3. The link-rails are designed to contact with rollers and support the machine weight. It also contacts the idlers and carrier rollers that provide guidance to the track motion. The track rail doesn't have fixed geometry and the rail width varies. The contact detection algorithm should account for this variation during simulation. The pin-bushing box connects track-link to its neighboring links. The track shoe is the part that provides the traction forces from the interaction with the ground through the grousers. The number of grousers in the track shoe depends on the machine type and the application. In general:

- Single grouser shoes: are used for dozers where grip and traction are the main factors.

- Double grouser shoes: are used for tracked loaders where medium traction and maximum maneuverability are needed.

- Triple grouser shoes: are used for excavators where a firm grip, low ground pressure and maneuverability are needed.

For simplicity, each chain unit (the two rails and shoe) will be referred to as track-link. The two rails are typically connected with bushing from the front side while a pin is inserted inside the bushing to connect it to the following link, as shown in Figure 3. The pin and bushing are designed and sealed to enclose lubricant that reduces friction during operation. The track-links typically contact the roller and idlers to distribute the machine load on the ground. The high contact forces induce wear in the track chain components and the contacting bodies. The wear rate and wear profile depends on the magnitude and distribution of the forces and on the relative velocity between the contacting bodies (Johnson, 1994) and (Strong, 2004). Accurate prediction of the contact force and relative velocity at the contact point is crucial for accurate wear prediction.

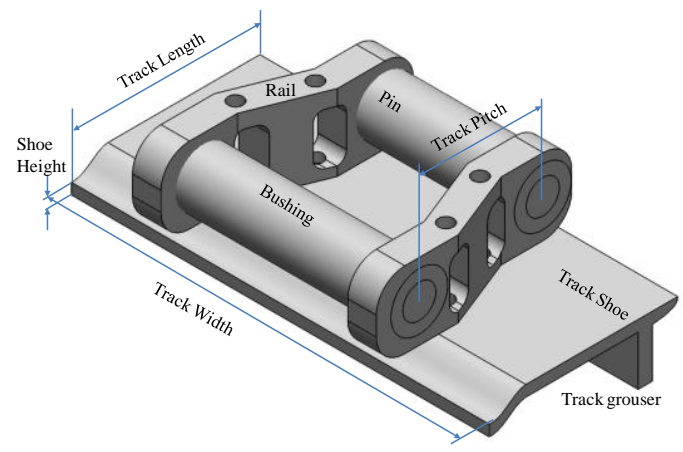

Figure 3. Track-link assembly box: single grouser shoe and track-link 
The dynamic equations of motion of the main system (upper chassis, undercarriage, and tooling mechanism) could be expressed using any general purpose multibody dynamics formulation. For example, the joint coordinates based approach and the spatial algebra operator could be used to express the system equations of motion as follows (Featherstone, 2008) and (Omar, 2014):

$$
\left[\begin{array}{ccc}
M^{\ell \ell} & 0 & \left(T_{a}^{\ell \ell}\right)^{T} \\
0 & 0 & -\mathbf{H}_{a}^{\ell} \\
T_{a}^{\ell \ell} & -\left(\mathbf{H}_{a}^{\ell}\right)^{T} & 0
\end{array}\right]\left[\begin{array}{c}
\mathbf{a}_{a}^{\ell} \\
\ddot{\mathbf{q}}_{a} \\
-\mathbf{F}_{a}^{\ell}
\end{array}\right]=\left[\begin{array}{c}
\mathbf{G}_{a}^{\ell}+\left(\tilde{\mathbf{v}}_{a}^{\ell}\right)^{T} \mathbf{P}_{a}^{\ell} \\
Q_{a} \\
\boldsymbol{\gamma}_{a}^{\ell}
\end{array}\right]
$$

where $M^{\ell \ell}$ is the block diagonal system inertia matrix, $T_{a}^{\ell \ell}$ is the joint connectivity or topology matrix for the system, $\mathbf{H}_{a}^{\ell}$ the joint partial velocity coefficients matrix, $\mathbf{a}_{a}^{\ell}$ vector of Cartesian spatial accelerations, $\mathbf{q}_{a}, \dot{\mathbf{q}}_{a}$, and $\ddot{\mathbf{q}}_{a}$ vector of joint coordinates and derivatives, $\mathbf{F}_{a}^{\ell}$ vector of joint reaction forces, $\mathbf{G}_{a}^{\ell}$ vector of applied spatial forces acting on the system, $\tilde{\mathbf{v}}_{a}^{\ell}$ the spatial cross-product matrix associated with vector of spatial velocities, $\mathbf{P}_{a}^{\ell}$ the momentum of all the bodies, $Q_{a}$ the applied loads in the joint space, and $\gamma_{a}^{\ell}$ is the quadratic velocity vector. The system of equations in (1) contains the vector of global acceleration and the vector of second derivatives of all the joint variables in the system. Since the inertia matrix $M^{\ell}$ is invertible, equation (1) could be solved to get the unknown joint accelerations $\ddot{\mathbf{q}}_{a}$ leading to the minimum set of dynamic equations as follows, starting by computing the Cartesian accelerations:

$$
\mathbf{a}_{a}^{\ell}=\left(T_{a}^{\ell \ell}\right)^{-1} \mathbf{H}_{a}^{\ell} \ddot{\mathbf{q}}_{a}+\left(T_{a}^{\ell \ell}\right)^{-1} \boldsymbol{\gamma}_{a}^{\ell}
$$

The joint reaction forces can be solved for as follows

$$
\mathbf{F}_{a}^{\ell}=\left(T_{a}^{\ell \ell}\right)^{-1} M^{\ell \ell} \mathbf{a}_{a}^{\ell}-\left(T_{a}^{\ell \ell}\right)^{-T}\left(\mathbf{G}_{a}^{\ell}+\left(\tilde{\mathbf{v}}_{a}^{\ell}\right)^{T} \mathbf{P}_{a}^{\ell}\right)
$$

The joint cartesian forces are projected into the joint subspace as follows:

$$
\mathbf{H}_{a}^{\ell} \mathbf{F}_{a}^{\ell}=Q_{a}
$$

The joint acceleration could be expressed in the following form:

$$
\begin{aligned}
& \left(\mathbf{H}_{a}^{\ell}\right)^{T}\left(T_{a}^{\ell \ell}\right)^{-T} M^{\ell \ell}\left(T_{a}^{\ell \ell}\right)^{-1} \mathbf{H}_{a}^{\ell} \ddot{q}_{a}=\mathbf{Q}_{a}+ \\
& \quad\left(\mathbf{H}_{a}^{\ell}\right)^{T}\left(T_{a}^{\ell \ell}\right)^{-T}\left(\left(\mathbf{G}_{a}^{\ell}+\left(\tilde{\mathbf{v}}_{a}^{\ell}\right)^{T} \mathbf{P}_{a}^{\ell}\right)-M^{\ell \ell}\left(T_{a}^{\ell \ell}\right)^{-1} \boldsymbol{\gamma}_{a}^{\ell}\right)
\end{aligned}
$$

The equation of motion could be written in a simple form as follows:

$$
M_{q q} \ddot{\mathbf{q}}_{a}=Q_{q}
$$

where $M_{q q}=\left(\mathbf{H}_{a}^{\ell}\right)^{T}\left(T_{a}^{\ell \ell}\right)^{-T} M^{\ell \ell}\left(T_{a}^{\ell \ell}\right)^{-1} \mathbf{H}_{a}^{\ell}$ is the projection of the apparent inertia matrix into the joint coordinate subspace $Q_{q}=\mathbf{Q}_{a}+\left(\mathbf{H}_{a}^{\ell}\right)^{T}\left(T_{a}^{\ell \ell}\right)^{-T}\left(\left(\mathbf{G}_{a}^{\ell}+\left(\tilde{\mathbf{v}}_{a}^{\ell}\right)^{T} \mathbf{P}_{a}^{\ell}\right)-M^{\ell \ell}\left(T_{a}^{\ell \ell}\right)^{-1} \gamma_{a}^{\ell}\right)$ is the projection of the Cartesian forces, centrifugal and Coriolis forces, and the gravity forces into the joint coordinate subspace.

The inertia matrix in equation (6) could be inverted and the joint accelerations could be computed. (Featherstone, 2008) and (Featherstone, 2005) demonstrated that the number of projections across the kinematic tree is highly dependent on the number of joint degrees of freedom between the child and root body, $n_{\text {list }}$ in the system kinematic tree. The number of operation is in the order of $\left(n_{l i s t}\right)^{3}$. For this reason, the depth of the kinematic tree should be minimized in order to 
improve the computational efficiency.

\section{Track-Link Kinematic and Dynamic Equations of Motion}

In order to reduce the problem dimensionality and develop a general purpose independent algorithm, the track links will be decoupled from the kinematic tree of the main system. All the track links can be considered as floating bodies and referenced directly to the inertial frame with 6-DOF joint and called floating bodies (FB). This means, the track links are not part of the kinematic tree and the topology matrix doesn't need to include any of their connectivity information. For this reason, track links will not project any inertia into any bodies in the main system and their inertia will not be affected by any decedent bodies. FB representing chain links will be interacting with other system components through compliant force elements: contact with sprocket, contact of the rollers and idlers with guide rails, and connection to the neighboring links. These contact forces can be calculated in the global coordinate system, as will be shown in the following sections, then will be transformed into the local link reference CS. Since all external force vectors are calculated and accumulated in the global CS, it can be then transformed once to the FB local CS. Also, there will be no need to transform the FB mass matrix to the inertial CS each time step. This approach significantly improves the computational efficiency by reducing the depth of the kinematic tree.

The position vector of the link $i$ reference frame could be defined with respect to an inertial reference frame using the vector $\underline{\mathbf{r}}_{o}^{i}=\left[\begin{array}{ccc}x^{i} & y^{i} & z^{i}\end{array}\right]^{T}$ while the body orientation could be defined by a vector of Euler parameters $E^{i}=\left[\begin{array}{llll}e_{0}^{i} & e_{1}^{i} & e_{2}^{i} & e_{3}^{i}\end{array}\right]^{T}$. The global velocity vector can be obtained by differentiating the position vector with respect to time. The spatial velocity vector of the track-link can be represented by a combination of two $3 x 1$ vectors: $\underline{\omega}$ represents the angular velocity of the track-link and $\underline{\dot{\mathbf{r}}}_{o}$ represents the linear velocity of the origin of the link's reference frame at point, $O$, (Featherstone, 2008), (Omar, 2014). The spatial velocity vector of link $i$ could be written as:

$$
\mathbf{v}^{i}=\left[\begin{array}{ll}
\underline{\omega} & \dot{\underline{r}}_{o}
\end{array}\right]^{T}
$$

The global angular velocity of the link could be obtained from derivatives of its Euler parameters as follows (Shabana, 2001):

$$
\left[\begin{array}{c}
\omega_{1} \\
\omega_{2} \\
\omega_{3}
\end{array}\right]=2\left[\begin{array}{cccc}
-e_{1} & -e_{0} & -e_{3} & e_{2} \\
-e_{2} & e_{3} & e_{0} & -e_{1} \\
-e_{3} & -e_{2} & e_{1} & e_{0}
\end{array}\right]\left[\begin{array}{c}
\dot{e}_{0} \\
\dot{e}_{1} \\
\dot{e}_{2} \\
\dot{e}_{3}
\end{array}\right]
$$

The spatial velocity expressed in coordinate system (CS) at $j$ can be transformed into any CS $i$ through the spatial transformation matrix as follows (Featherstone, 2008), 0(Omar, 2014):

$$
\mathbf{v}^{i}=X^{i j} \mathbf{v}^{j}
$$

where $X^{i j}$ is the spatial transformation matrix, $i$ and $j$ indicate the coordinate systems. The spatial transformation matrix $X^{i j}$ is the result of rotation followed by a translation as follows:

$$
X^{i j}=L^{i j} R^{i j}=\left[\begin{array}{cc}
I & 0 \\
\tilde{\tilde{r}}_{i j}^{i} & I
\end{array}\right]\left[\begin{array}{cc}
\underline{R}^{i j} & 0 \\
0 & \underline{R}^{i j}
\end{array}\right]=\left[\begin{array}{cc}
\underline{R}^{i j} & 0 \\
\underline{R}^{i j} \tilde{r}_{i j}^{i j} & \underline{R}^{i j}
\end{array}\right]
$$

where $L^{i j}$ is the $6 \times 6$ spatial translation matrix, $\underline{\underline{r}}_{i j}^{i}$ is the $3 \times 1$ translational displacement vectors of CS $j$ relative to CS $i$

defined in the CS $i, \quad \underline{\underline{r}}_{i j}^{i}$ is $3 \times 3$ skew symmetric matrix representing the cross-product operation of $\underline{r}_{i j}^{i}, R^{i j}$ is the 6x6 spatial rotation matrix, $\underline{R}^{i j}$ is the $3 \times 3$ transformation matrix relating the coordinate frames $i$ and $j$. It should be noted 
that the general spatial transformation matrix $X^{i j}$ is not orthonormal. The spatial velocity vector $\mathbf{v}_{i j, k}^{i}$ is defined in a specific coordinate system $i$ (superscript $i$ ) measuring the velocity difference between two markers $i$ and $j$ (subscript $i j$ ) and is defined with respect to a certain observer located at $k$ (subscript after comma $k$ ). The relative spatial velocity vector $\mathbf{v}_{i j, k}^{i}$ between body $i$ and body $j$ defined in CS of body $j$ observed at the origin of $k$ can be written as follows:

$$
\mathbf{v}_{i j, k}^{j}=\left[\begin{array}{c}
\omega_{i j}^{j} \\
\underline{\dot{r}}_{i j, k}^{j}
\end{array}\right]
$$

where $\underline{\omega}_{i j}^{j}$ is the time derivative of the orientation variables between CS $i$ and CS $j$ defined in CS $j$ and $\underline{\dot{r}}_{i j, j}^{j}$ is the translational velocity between CS $i$ and $\operatorname{CS} j$.

The equation of motion of any link in the track chain could be obtained by differentiating its vector of spatial momentum with respect to time. The spatial inertia matrix $M_{j, G}$ of link $j$ defined at the link's center of mass marker $G$, can be written as follows:

$$
M_{j, G}=\left[\begin{array}{cc}
\underline{J}_{j, G} & 0 \\
0 & m_{j} \underline{I}
\end{array}\right]
$$

where $m_{j}$ is the link mass, $\underline{I}$ is $3 \times 3$ identity matrix and $\underline{J}_{j, G}$ is the $3 \times 3$ matrix representing the link $j$ moment of inertia defined at the marker located at the link center of mass, $G$. The spatial momentum vector $\mathbf{P}_{j, G}^{j}$ could be calculated as follows:

$$
\mathbf{P}_{j, G}^{j}=M_{j, G} \mathbf{v}_{0 j, G}^{j}
$$

In order to obtain the equation of motion, the spatial momentum should be expressed in the global coordinate system as follows:

$$
\mathbf{P}_{j, 0}^{0}=M_{j, 0} \mathbf{v}_{0 j, 0}^{0} \quad \text { where } M_{j, 0}=X^{0 j-T} M_{j, j}^{j} X^{0 j-1}=X^{j 0 T} M_{j, j} X^{j 0}
$$

Differentiating the momentum equation will lead to the link's equation of motion as follows:

$$
M_{j, 0} \mathbf{a}_{0 j, 0}^{0}-\tilde{\mathbf{v}}_{0 j, 0}^{0 T} M_{j, 0} \mathbf{v}_{0 j, 0}^{0}=\mathbf{G}_{j, 0}^{0}
$$

where $\mathbf{G}_{j, 0}^{0}=\left[\begin{array}{cc}\underline{\tau}_{j, 0}^{0} & \underline{g}_{j}^{0}\end{array}\right]^{T}$ is the spatial force vector including the gravitational forces, $\underline{\tau}_{j, 0}^{0}$ is the sum of all external torques acting on the body and $\underline{g}_{j}^{0}$ is the sum of all external forces acting on the body.

The equation of motion of FB in local CS can be written as follows:

$$
M_{j, j} \mathbf{a}_{0 j, j}^{j}=\mathbf{G}_{j, j}^{j}+\tilde{\mathbf{v}}_{0 j, j}^{j T} M_{j, j} \mathbf{v}_{0 j, j}^{j}
$$

The link mass matrix can be easily inverted and the link accelerations could be directly integrated as follows:

$$
\mathbf{a}_{0 j, j}^{j}=\left(M_{j, j}\right)^{-1}\left(\mathbf{G}_{j, j}^{j}+\tilde{\mathbf{v}}_{0 j, j}^{j T} M_{j, j} \mathbf{v}_{0 j, j}^{j}\right)
$$


Since each FB is referenced with 6-DOF, the rotational transformation matrix need to be updated using the body rotational parameters. In the proposed approach, Euler parameters are used describe the orientation of the FB and that requires using additional constraint equation. It was reported by many researchers (McCullough et al 1985), (Roberson \& Ming-Ming, 1986), (Omar, 2014) that in the case of long chain or track systems, the Euler parameters norm in the integrator may drift from unity causing phase shift. In order to improve the computational efficiency, the constraint equation could be eliminated and replaced with error penalty feedback within the integrator. Assuming Euler parameters are defined as $E=\left[\begin{array}{llll}e_{0} & e_{1} & e_{2} & e_{3}\end{array}\right]^{T}$ and $E_{s}=\left[\begin{array}{lll}e_{1} & e_{2} & e_{3}\end{array}\right]^{T}$. The normalized Euler parameters can be calculated as follows:

$$
\underline{E}_{s}=E_{s} / \sqrt{E_{s}^{T} E_{s}}
$$

The error in calculating Euler parameters can be approximated as:

$$
E_{e r r}=1-\left|\underline{E}_{s}\right|
$$

This error may be fed back into the Euler parameter differential equations as

$$
\underline{\dot{E}}_{s} \Leftarrow \underline{\dot{E}}_{s}-2 E_{e r r} \underline{E}_{s}
$$

Equation (20) shows that a small negative correction, proportional to error and the Euler parameters, is fed back into the differential equations. Implementing the feedback term into (20) will normalize the Euler parameters without introducing constraint equations to the system.

\section{Track-System Interaction}

This section presents three modules necessary to model the interaction forces in the super-component.

\subsection{Link Pin-Bushing Force Module:}

The track-links connected are connected by pin-bushing element which transmits the traction forces throughout the track links. The pin-bushing could be subjected to tensile load due to the traction forces, bending loads due to track twisting and shear forces due to sideway force and aligning moments. In this proposed approach, a compliant bushing force element is used to model the connection between two consecutive links. Other approaches for modeling joints with clearances could be found in the literatures (Flores et al 2004) and (Pereira et al 2011). The track-links are designed to allow small relative back-bending. When back-bending of the track links becomes excessive, a contact force between the adjacent shoe surfaces is developed to prevent damaging the links. The force module under consideration accounts for the elastic and back-bending contact forces. The kinematic diagram the link's pin-bushing force element connecting two link-boxes is shown in Figure 4.

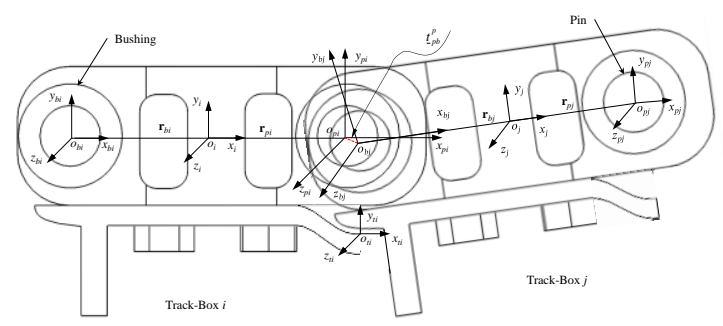

Figure 4. Kinematic description of the pin-bushing element in the track link-box

Considering two consecutive link-boxes, $i$ and $j$, where each link-box has two markers to locate the pin and bushing centers with respect to the link reference marker. $O_{p i}$ and $O_{b i}$ locate the center of the pin and bushing of link-box $i$ with respect to the frame of reference, $O_{i}$, while $O_{p j}$ and $O_{b j}$ locate the centers of the pin and bushing of link-box $j$ with respect to the reference frame of link-box, $O_{j}$. Each pin marker of the link-box is connected to the bushing marker of the neighboring link via a bushing force element. The pin and bushing markers connected by the bushing element are considered to be coincident and parallel if there is no deflection or relative rotation between links, as shown in Figure 4 . In order to calculate the bushing forces, the translational deflection and relative orientation between the pin and bushing 
marker should be calculated and updated every time step. During the dynamic simulation, the position and velocity vectors of each body's reference frame are calculated. Also, the pin and bushing global position vectors, $\underline{r}_{0 p}^{0}$ and $\underline{r}_{0 b}^{0}$, and the global transformation matrices, $\underline{R}^{0 p}$ and $\underline{R}^{0 b}$, can be calculated using Equations (10). The relative displacement vector between the two markers and the orientation matrix could be calculated in the pin coordinate system as follows.

$$
\underline{r}_{p b}^{0}=\underline{r}_{0 b}^{0}-\underline{r}_{0 p}^{0}, \underline{r}_{p b}^{p}=\left(\underline{R}^{0 p}\right)^{-1} \underline{r}_{p b}^{0}, \quad \text { and } \quad \underline{R}^{p b}=\left(\underline{R}^{0 p}\right)^{-1} \underline{R}^{0 b}
$$

Once transformed into the pin coordinate system, the bushing deformation is represented by the relative translational displacement vector $\underline{r}_{p b}^{p}$, and the relative orientation matrix $\underline{R}^{p b}$. The bushing rotational deformation can be calculated in two steps from orientation matrix as follows. Using the relative orientation matrix $\underline{R}^{p b}$, Euler parameters can be calculated by expressing the transformation matrix in terms of Euler parameters as follows, (Shampine, 1975) and (Shabana, 2001):

$$
\underline{R}^{p b}=\underline{I}+2 \underline{E}_{s}\left(e_{0} \underline{I}+\underline{E}_{s}\right), \quad \underline{E}_{s}=\left[\begin{array}{lll}
e_{1} & e_{2} & e_{3}
\end{array}\right]^{T}
$$

Then Euler parameters can be converted into a rotational angle $\theta$ around a vector $\underline{u}^{r}$ as follows, (Roberson et al 1986) (Omar, 2014):

$$
\begin{aligned}
& e_{0}=\cos (\theta / 2), \\
& e_{1}=u_{1}^{r} \sin (\theta / 2), \\
& e_{2}=u_{2}^{r} \sin (\theta / 2), \\
& e_{3}=u_{3}^{r} \sin (\theta / 2)
\end{aligned}
$$

The relative velocity vector between the pin and bushing markers can be calculated and transformed into the pin coordinate frame as follows:

$$
\mathbf{v}_{0 b, p}^{0}=\left[\begin{array}{cc}
I & 0 \\
\underline{\tilde{r}}_{0 p}^{0}-\underline{\tilde{r}}_{0 b}^{0} & I
\end{array}\right]\left[\begin{array}{c}
\omega_{0 b}^{0} \\
\underline{t}_{0 b, b}^{0}
\end{array}\right], \quad \mathbf{v}_{p b, p}^{p}=\left(X_{0 p}^{0 p}\right)^{-1}\left(\mathbf{v}_{0 b, p}^{0}-\mathbf{v}_{0 p, p}^{0}\right) \quad \text { and } \quad \mathbf{v}_{p b, p}^{p}=\left[\begin{array}{c}
\omega_{p b}^{p} \\
\underline{\dot{r}}_{p b, p}^{p}
\end{array}\right]=\left[\begin{array}{cc}
I & 0 \\
\tilde{r}_{p b}^{p} & I
\end{array}\right]\left[\begin{array}{c}
\omega_{p b}^{p} \\
\underline{\dot{r}}_{p b, p}^{p}
\end{array}\right]
$$

It is assumed that the bushing element produces three force components $F_{x}, F_{y}$, and $F_{z}$ that are functions of the relative displacements and velocities between the pin and bushing markers; and two torque components $T_{x}$, and $T_{y}$, that are functions of the relative angular rotations and angular velocities between the markers. The torque component $T_{z}$ is assumed to be zero in order to allow relative rotation. The bushing forces and torques can be computed in the pin marker coordinate system as follows:

$$
F_{p, p}^{p}=\left[\begin{array}{c}
-\left(\theta K_{t x}\right) u_{1}^{r}-\omega_{x} C_{t x} \\
-\left(\theta K_{t y}\right) u_{2}^{r}-\omega_{y} C_{t y} \\
-\left(\theta K_{t z}\right) u_{3}^{r}-\omega_{z} C_{t z} \\
-t_{x} K_{x}-\dot{t}_{x} C_{x} \\
-t_{y} K_{y}-\dot{t}_{y} C_{y} \\
-t_{z} K_{z}-\dot{t}_{z} C_{z}
\end{array}\right]
$$

where $K_{t x}, K_{t y}, K_{t z}, C_{t x}, C_{t y}$, and $C_{t z}$ represent the rotational stiffness and damping coefficients $K_{x}, K_{y}, K_{z}, C_{x}, C_{y}$, and $C_{z}$, represent the translational stiffness and damping coefficients. The value of $K_{t z}$ could be set to zero to model free rotation.

If the track links are bent backward, a metal-to-metal contact occurs between the two track-shoes of the two adjacent links leading to large contact forces. The interaction contact forces between the track link-boxes need to be accounted for. The proposed force module follows two approaches to detect the contact: first approach is based on the back-bending angle if it exceeded certain preset angle, the contact forces are applied. The preset angle is defined based on the track and shoe design parameters: shoe thickness, length, the chamfer radius, and other geometrical features. The second approach of contact detection is based on the geometry of the links and that is more computationally demanding. 
The forces and torques could now be transformed into the bushing marker coordinate system. The forces and moments from the bushing force element could then be applied to the link's equation of motion (16). The bushing element stiffness coefficients can be calculated experimentally of using a finite element model for the pin-link and bushing link while the damping coefficient could be chosen to represent the structural damping.

\subsection{Link-Sprocket Contact}

In tracked vehicles, rotary motion of the sprocket is transformed into translational motion of the track-links and at the same time power is transferred through contact between the track-links' bushings with the sprocket teeth. As shown in Figure 5, the sprocket teeth could be engaged with multiple link-bushings at the same time. Due to the relative motion between the track-links and the sprockets, the contact point between the sprocket tooth and the bushing slides along the sprocket tooth surface. The magnitude of the contact force changes at the different positions as the sprocket profile has different curvatures. Also, the contact could occur in the front or the back of the sprocket tooth depending on forward or backward driving. In many existing commercial codes, the contact between the sprocket teeth and bushing is simplified contact models. Approximated cylinder-to-cylinder contact or sphere-to-cylinder contact are commonly used for contact detection. The resulting forces are not accurate for the design and optimization purposes. Although most of the track motion is apparently planar, contact between the surface of the sprocket teeth and the bushing cylinder could be considered planar too. However, due to the lateral forces and out-of-plan motion/vibration, contact may occur between the sprocket tooth and the sides of the track links.

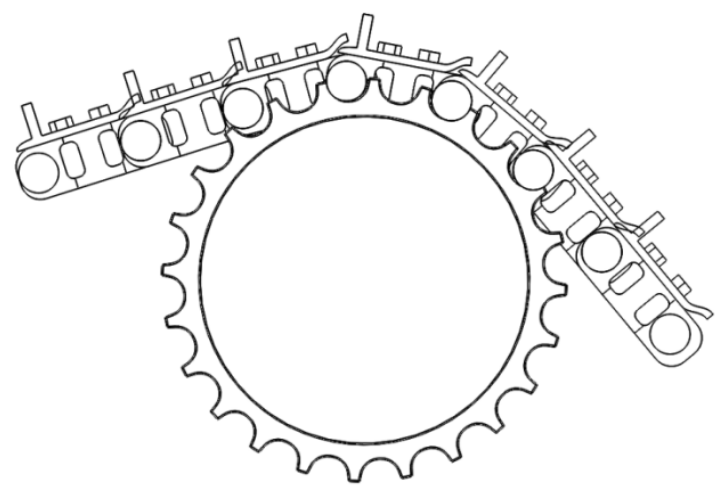

Figure 5. Sprocket teeth in contact with the link bushings

The sprocket tooth could be described by detailed geometric features, $x-y$ spline curve or approximated using tangent lines. The first two approaches are computationally intensive while the last approach leads to reasonably accurate results faster (Gottschalk et al 1996) and (Omar, 2014). In this paper, the tooth profile is approximated using six points defined in the $x-y$ plane relative to the sprocket center, as shown Figure 6 . The tooth profile will be replicated around $z$-axis according to the number of sprocket teeth. The tooth thickness could be specified to fully define the sprocket geometry and to detect the side contact with the link rails. Based on the approximated profile points and the sprocket thickness, five possible contact surfaces could be defined. In order to improve the computational efficiency during run time, the profile-tangent and -normal vectors could be determined for each surface segment prior to the simulation and saved for repeated calculations.

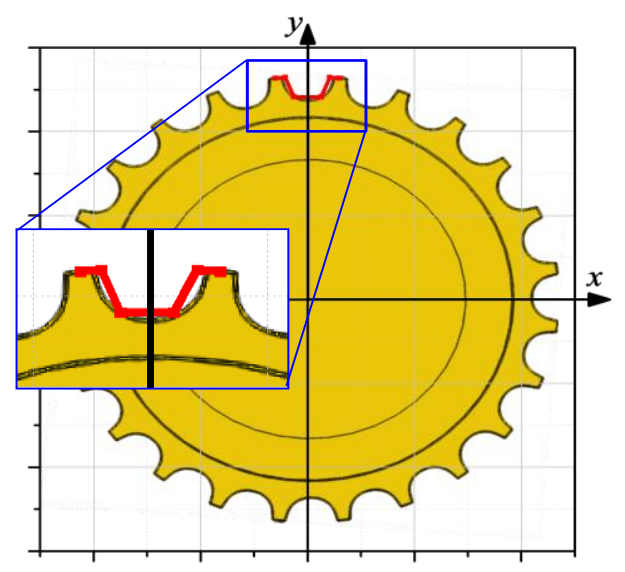

Figure 6. Approximated sprocket tooth profile 
The contact detection algorithm loops over all the sprockets in the system to identify the track-links that are within a pre-specified proximity range of each sprocket. The proximity range could be set as the sprocket outer radius plus one track-link pitch. Referring to Figure 7, the relative position vector of the link reference frame with respect to the sprocket frame reference could be defined in the sprocket reference frame as follows:

$$
\underline{r}_{s l}^{0}=\underline{r}_{0 l}^{0}-\underline{r}_{0 s}^{0}, \underline{t}_{s l}^{s}=\left(\underline{R}^{0 s}\right)^{-1} \underline{t}_{s l}^{0}, \text { and } \underline{R}^{s l}=\left(\underline{R}^{0 s}\right)^{-1} \underline{R}^{0 l}
$$

where $\underline{\underline{x}}_{0 s}^{0}$ and $\underline{r}_{0 l}^{0}$ is the global position vector of the sprocket and the link reference frames, $\underline{r}_{s l}^{0}$ and $\underline{r}_{s l}^{s}=\left[\begin{array}{lll}r_{x} & r_{y} & r_{z}\end{array}\right]^{T}$ are the relative position vectors in the global frame and in the local sprocket frame, $\underline{R}^{0 s}$ and $\underline{R}^{0 l}$ are the global orientation matrix of the sprocket and bushing frames, $\underline{R}^{s l}$ is the relative orientation matrix between the sprocket and the link. It should be mentioned that the sprockets position vectors and orientation matrices are passed to the contact detection module from the main code while the track- links position vector and orientation matrices are calculated within the proposed super-component. The distance $d$ between the sprocket center and the link reference frame is computed as:

$$
d=\sqrt{\left(r_{x}^{2}+r_{y}^{2}\right)}
$$

If the distance $d$ is less than the pre-specified proximity, $\delta_{\text {prox }}$, then contact might occur between this link and the sprocket teeth profile. In order to exactly identify the contact point, the position vector of the bushing center is calculated and transformed into the sprocket plane as follows:

$$
\underline{r}_{s b}^{0}=\underline{r}_{0 b}^{0}-\underline{r}_{0 s}^{0}, \quad \underline{r}_{s b}^{s}=\left(\underline{R}^{0 s}\right)^{-1} \underline{r}_{s b}^{0}, \quad \text { and } \quad \underline{R}^{s b}=\left(\underline{R}^{0 s}\right)^{-1} \underline{R}^{0 b}
$$

where $\underline{r}_{0 s}^{0}$ and $\underline{r}_{0 b}^{0}$ is the global position vector of the sprocket and the bushing marker, $\underline{\underline{r}}_{s b}^{0}$ and $\underline{\underline{r}}_{s b}^{s}=\left[\begin{array}{lll}r_{x} & r_{y} & r_{z}\end{array}\right]^{T}$ are the relative position vector in global and in the sprocket frame, $\underline{R}^{0 s}$ and $R^{0 b}$ is the global orientation matrix of the sprocket and bushing frames, and $\underline{R}^{s b}$ is the relative orientation matrix between the sprocket and the bushing. The components of the relative position vector could be used to determine the angle $\psi$ between the relative position vector and the vertical $y$-axis of the sprocket reference frame and hence the contact tooth index could be identified. The relative position between the profile points to bushing center is determined and lines $L_{1.6}$ are defined, as shown in Figure 7. Contact can occur between the bushing circle and the profile line segment if the projection of the tangent vector passes through the center of the circle or if either segment end-point is falls inside the circle. The normal distance between the bushing center and the contact profile segment could be easily determined and compared to the bushing radius, $R_{b}$, to determine if penetration occurred. The position vector of exact contact point, $p_{c}$, relative to the sprocket reference frame of the could determined as $\underline{r}_{s p}^{s}$, and the angle $\varphi$ could be calculated from the vector coordinates.

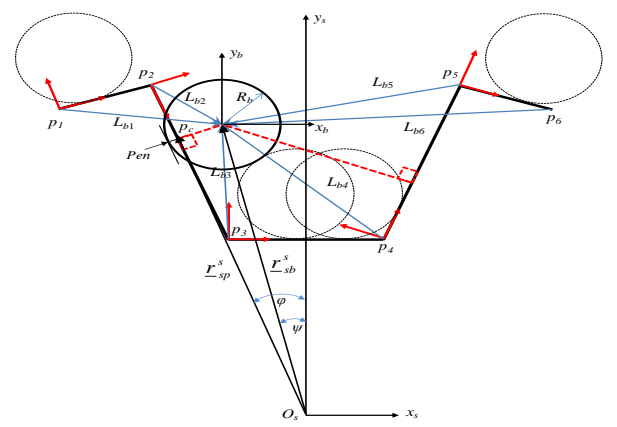

Figure 7. Bushing-sprocket contact detection 
The global velocity of the bushing link and sprocket, $\mathbf{v}_{0 b, b}^{0}$ and $\mathbf{v}_{0 s, s}^{0}$, are computed every time step. The relative velocity between track-link and sprocket can be computed as follows. The bushing marker velocity is computed at the sprocket reference frame:

$$
\mathbf{v}_{0 b, s}^{0}=\left[\begin{array}{cc}
I & 0 \\
\underline{\tilde{r}}_{0 b}^{0}-\underline{\tilde{r}}_{0 s}^{0} & I
\end{array}\right]\left[\begin{array}{c}
\omega_{0 b}^{0} \\
\underline{\dot{r}}_{0 b, b}^{0}
\end{array}\right]
$$

The relative velocity can be computed in global at the sprocket reference frame as follows:

$$
\mathbf{v}_{s b, s}^{s}=\left(X_{0 s}^{0 s}\right)^{-1}\left(\mathbf{v}_{0 b, s}^{0}-\mathbf{v}_{0 s, s}^{0}\right)
$$

The relative velocity between the bushing marker and the sprocket at the contact point could be computed as follows:

$$
\mathbf{v}_{s b, p}^{s}=\left[\begin{array}{c}
\omega_{s b}^{s} \\
\dot{\underline{r}}_{s b, s}^{s}
\end{array}\right]=\left[\begin{array}{cc}
I & 0 \\
\underline{\tilde{r}}_{s p}^{s} & I
\end{array}\right]\left[\begin{array}{l}
\omega_{s b}^{s} \\
\dot{\underline{r}}_{s b, s}^{s}
\end{array}\right]
$$

In order to calculate the normal and sliding velocity components, $\underline{\dot{r}}_{s b, s}^{s}=\left[\begin{array}{lll}v_{r x} & v_{r y} & v_{r z}\end{array}\right]^{T}$ needs to be transformed using the $\varphi$ as follows:

$$
\left[\begin{array}{c}
v_{x-s l i d} \\
v_{\text {norm }} \\
v_{z-\text { slid }}
\end{array}\right]=\left[\begin{array}{ccc}
\cos \varphi & \sin \varphi & 0 \\
-\sin \varphi & \cos \varphi & 0 \\
0 & 0 & 1
\end{array}\right]\left[\begin{array}{l}
v_{r x} \\
v_{r y} \\
v_{r z}
\end{array}\right]
$$

The sliding velocity could be computed as:

$$
\underline{v}_{s}=\left|\underline{v}_{s}\right| \underline{u}_{v s} \quad \text { where } \quad\left|\underline{v}_{s}\right|=\sqrt{v_{x-s l i d}^{2}+v_{z-s l i d}^{2}} \quad \text { and } \quad \underline{u}_{v s}=\underline{v}_{s} /\left|\underline{v}_{s}\right|
$$

Several force models could be found in the literature (Johnson, 1994) and (Stronge, 2004). The normal contact force results from elastic spring force and damping force can be evaluated as follows:

$$
F_{n}=K \delta+C v_{n o r m}
$$

The sliding friction force is a function of the normal elastic force and the friction coefficient. Due to impact between the bushing and the sprocket, the normal force component could be significantly high while the sliding velocities are significantly small and that can lead to very large friction forces that can cause the simulation to fail. To reduce the effect of large friction force, the friction coefficient can be considered as smooth function of the sliding velocity as follows:

$$
\underline{F}_{f}=\mu S_{\mu}(K \delta) \underline{u}_{v s}, \text { where } \quad S_{\mu}=\min \left(1,2 \xi-\xi^{2}\right) \text { and } \xi=\left|\underline{v}_{s}\right| / v_{\mu}
$$

where $v_{\mu}$ is a predefined maximum sliding velocity at which the friction force reaches maximum value and $S_{\mu}$ is the friction force softening parameter (Johnson, 1994) and (Stronge, 2004). Other softening functions could be effectively used. The contact and friction forces vectors are rotated back to the sprocket reference frame as follows:

$$
\underline{F}^{c}=\left[\begin{array}{c}
F_{x} \\
F_{y} \\
F_{z}
\end{array}\right]=\left[\begin{array}{ccc}
\cos \varphi & -\sin \varphi & 0 \\
\sin \varphi & \cos \varphi & 0 \\
0 & 0 & 1
\end{array}\right]\left[\begin{array}{c}
F_{f x} \\
F_{n o r m} \\
F_{f z}
\end{array}\right]
$$


Then contact forces are now translated to the sprocket reference frame and the track-link reference frame as follows:

$$
F_{s, s}^{s}=\left[\begin{array}{cc}
I & 0 \\
\underline{\tilde{r}}_{s p}^{s} & I
\end{array}\right]^{-T}\left[\begin{array}{c}
0 \\
\underline{F}^{c}
\end{array}\right] \quad \text { and } \quad F_{l, l}^{l}=\left(X_{s l}^{s l}\right)^{-T} F_{s, s}^{s}
$$

It should be noted that contact can occur between the sprocket tooth and both the bushing cylinder and the link-rail sides. The contact algorithm should be able to detect both contacts and add the resulting forces and moments. It should be noted that the bushing cylinder is assumed to be rigid and will not deform under the effect of the contact forces.

\subsection{Track Chain Interaction with the Rollers and Idlers}

Carrier rollers, track rollers and idlers are geometrically similar components located on each side of the tracked vehicle, as shown in Figure 2, to provide guidance and ensure stability to the track motion during operation. The roller frame is one of the major undercarriage components that can carry the rollers and idlers. The carrier rollers are either connected to the vehicle chassis or the roller frame depending on the machine size and the track design. The carrier rollers are used to reduce flapping and back-bending of the track chain. Front track idlers are connected to the roller frame with a recoil mechanism while the rear idlers (if existed) are usually connected to the rear roller frame without recoil mechanism. The idlers and the recoil mechanism are very important to absorb the shock and impact forces and maintain the proper tension in the track. Track rollers are directly connected to the roller frame in small machines while in large machines road-arms are connected to the roller frame and each arm carries two track rollers. The track rollers are typically used to distribute the vehicle weight over the track links. The idler and rollers interact with all the track-link rail surfaces (top, outer and internal surfaces) and there is a potential to have multiple contact points simultaneously, as shown in Figure 8.
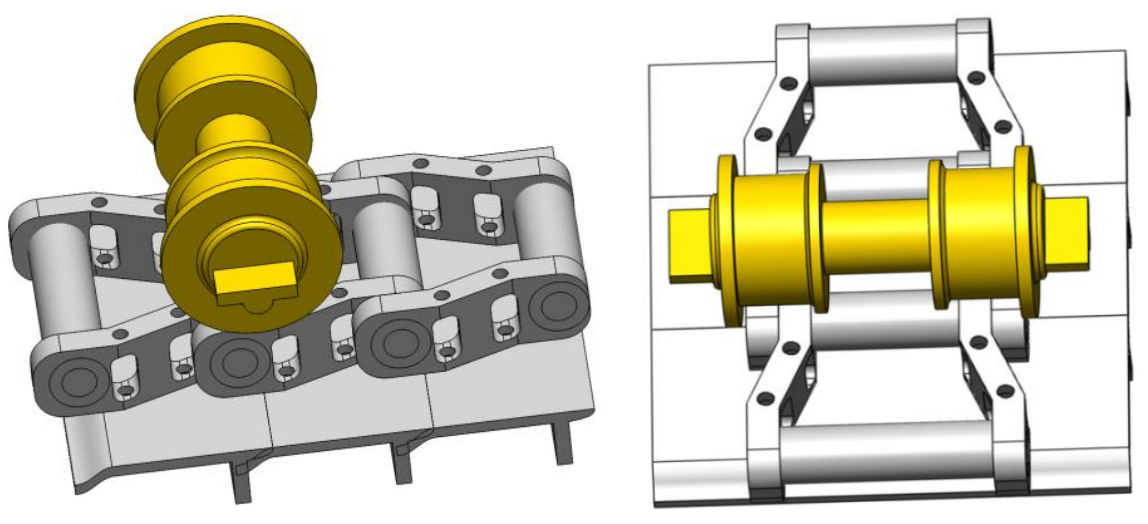

Figure 8. Track links and track roller showing potential of multiple link contact

In this proposed super-component approach a specialized contact algorithm is devised to account for all these potential contact forces. Most existing formulations approximate the roller by cylinders and the track-link by uniform surface.

Another source of complexity in detecting the track-roller contact is originated from the complex geometry of the track links contact surface, as shown in Figure 8. Due to the design requirements, the geometry of the rail surface is not uniform and varies along the longitudinal track axis. The rollers could be in contact with one or more links at the same time, as shown in Figure 8. It should be mentioned that contact detection between the roller and the track link is fundamentally similar to contact detection between the train wheel and the ground track. However in the case of roller-track, the contact is more complex because of the following reasons:

- In roller-track contact: the rail surface geometry varies and contact detection could be over any section while in train application, the rail has one uniform profile.

- The rollers and idlers have different profiles for the cross-sections while the train wheel has one uniform profile.

- In roller track contact occurs between two moving bodies while in the case of train contact the rail is stationary. 

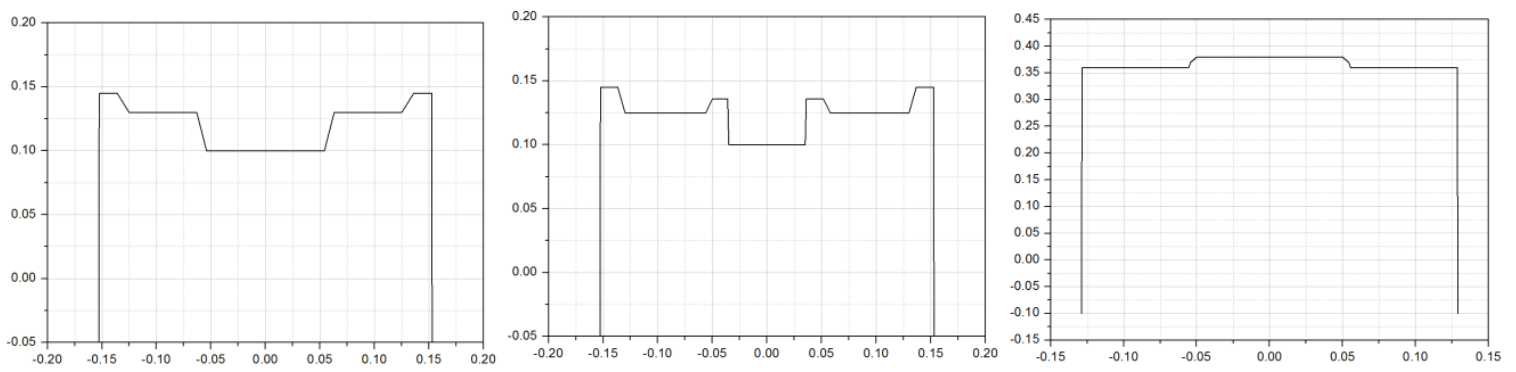

Figure 9. Spline representation of the cross-section of the carrier roller, track roller, and idler respectively

The proposed contact detection procedure is a complex quasi-3D model that utilizes different geometric description for each component. The module treats the roller, carrier and idler as surface of revolutions where the cross sections could described by a profile as shown in Figure 9. Since the track rail surface geometry changes, the rail surface is described at the different sections with a spline as shown in Figure 10. The cross-section splines are defined relative to the track link reference frame.

Contact detection in multibody system dynamics is one of the most time consuming tasks and any careful elimination of the redundant computations could result in significant improvement in the computational cost. Before the computation starts, a pre-processing step could be performed to reduce the computational over-head during the runtime. Using the roller profile data, the preprocessor can determine the maximum radius at each cross-sectional for each roller and identify the dimensions of the proximity box of each particular roller. Using the rail profile, defined as shown in Figure 10 , the preprocessor constructs a map for the link rail at each section.

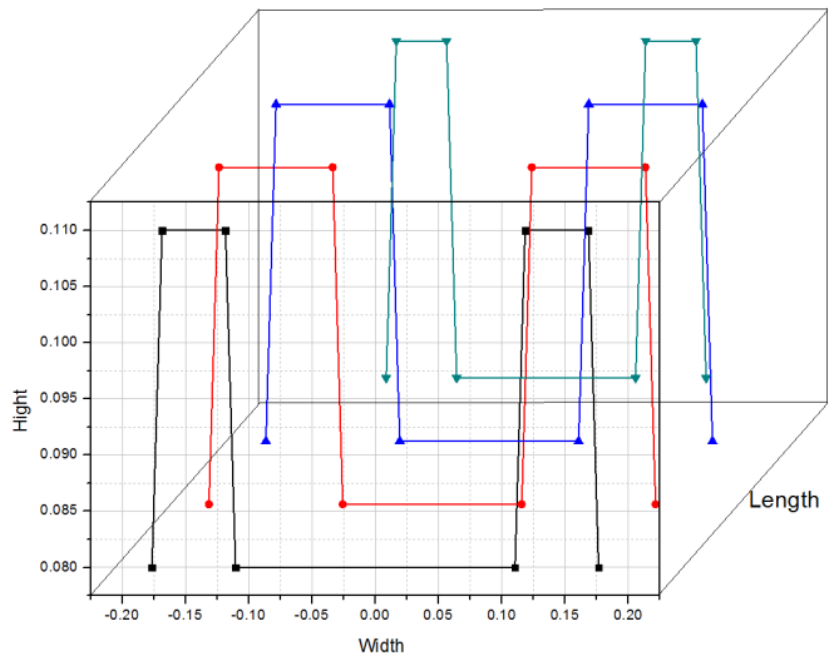

Figure 10. Track links surface geometry

During the simulation, the contact detection algorithm works in a similar fashion to the sprocket algorithm. The contact detection algorithm loops over all the carriers, rollers and idlers and identifies the track-links that are within its proximity and have potential for contact using boxing algorithm. A box is established around each roller with length $w_{b}=P_{\text {track }}+R_{\text {max }}$ and height $h_{b}=h_{\text {rail }}+R_{\max }$ where $P_{\text {track }}$ is the equal to the track pitch, $R_{\text {max }}$ is the roller maximum radius, and $h_{\text {rail }}$ is the track rail height. The box dimensions will ensure that more than one link will be within the contact proximity of the roller and available for contact detection for several simulation steps before re-boxing. This approach improves the computational efficiency. The following discussion focuses on the track roller and can be applied to the carrier roller and the idler as well.

Referring to Figure 11, the relative position vector of the link reference frame with respect to the roller frame reference could be defined in the sprocket reference frame as follows:

$$
\underline{r}_{r l}^{0}=\underline{r}_{0 l}^{0}-\underline{r}_{0 r}^{0}, \quad \underline{t}_{r l}^{r}=\left(\underline{R}^{0 r}\right)^{-1} \underline{t}_{r l}^{0}, \quad \text { and } \quad \underline{R}^{r l}=\left(\underline{R}^{0 r}\right)^{-1} \underline{R}^{0 l}
$$


where $\underline{r}_{0 r}^{0}$ and $\underline{r}_{0 l}^{0}$ are the global position vectors of the roller and the link reference frames respectively, $\underline{\underline{r}}_{r l}^{0}$ and $\underline{r}_{r l}^{r}=\left[\begin{array}{lll}r_{x} & r_{y} & r_{z}\end{array}\right]^{T}$ are the relative position vectors in the global frame and in the local roller frame, $\underline{R}^{0 r}$ and $\underline{R}^{0 l}$ are the global orientation matrix of the roller and bushing frames, $\underline{R}^{r l}$ is the relative orientation matrix between the roller and the link. The contact algorithm checks coordinates of the relative position vector against the box dimensions of the current roller, if the any of the links moved outside the box, re-boxing is considered. After the re-boxing check, the algorithm detects the contact between the roller and the links within its proximity.

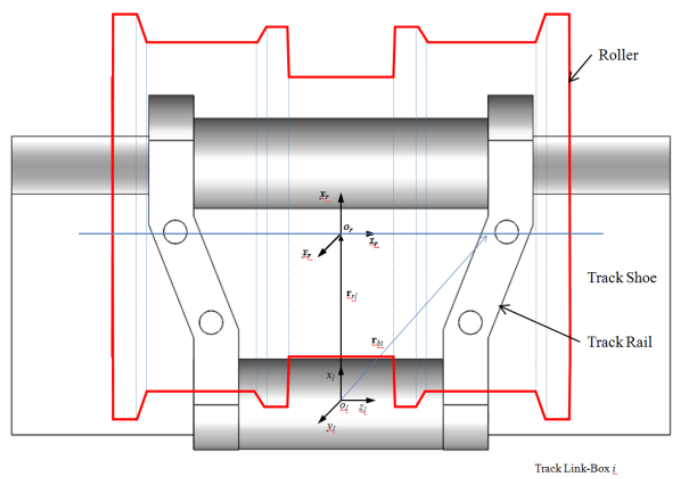

Figure 11. Kinematic contact between the roller and the track link.

As shown in Figure 10, the rail surface of the link is defined by profiles with respect to a certain origins along the local longitudinal $x$-axis of the track link coordinate system. In order to determine which rail section is in contact with the roller, the relative position vector, $\underline{r}_{r l}^{r}$, is projected into the link coordinate system as follows:

$$
\underline{r}_{r l}^{l}=\left(\underline{R}^{r l}\right)^{-1} \underline{r}_{r l}^{r}
$$

Using the coordinates of $\underline{r}_{r l}^{r}$ and the geometric description of the profiles, the contact section could be identified.

Once the contact section is identified, the normal and tangent to each line segment of the profile could be calculated in the local link coordinate system. Also, the profile direction should be identified, convex or concave. The roller profile is rotated into the local link coordinate system and the contact algorithm loops over the line segments of the roller profile and check its intersection with the line segments on the link profile. The position and penetration of all the contact points is calculated and stored for force application. It should be noted that this analysis has limitation in handling roller yaw relative to the rail.

The global velocity of the track link and roller, $\mathbf{v}_{0 b, b}^{0}$ and $\mathbf{v}_{0 s, s}^{0}$, are calculated every time step. The relative velocity between track link and roller can be computed as follows. The link velocity is computed at the roller reference frame:

$$
\mathbf{v}_{0 l, r}^{0}=\left[\begin{array}{cc}
I & 0 \\
\underline{\tilde{r}}_{0 l}^{0}-\underline{\tilde{r}}_{0 r}^{0} & I
\end{array}\right]\left[\begin{array}{c}
\omega_{0 l}^{0} \\
\underline{\underline{r}}_{0 l, l}^{0}
\end{array}\right]
$$

The relative velocity can be computed in global at the roller reference frame as follows:

$$
\mathbf{v}_{r l, r}^{r}=\left(X_{0 r}^{0 r}\right)^{-1}\left(\mathbf{v}_{0 l, r}^{0}-\mathbf{v}_{0 r, r}^{0}\right)
$$

The relative velocity between the link and the roller at the contact point, $p$, could be computed as follows: 


$$
\mathbf{v}_{r l, p}^{r}=\left[\begin{array}{c}
\omega_{r l}^{r} \\
\dot{\underline{r}}_{r l, r}^{r}
\end{array}\right]=\left[\begin{array}{cc}
I & 0 \\
\tilde{\tilde{r}}_{r p}^{r} & I
\end{array}\right]\left[\begin{array}{c}
\omega_{r l}^{r} \\
\dot{\underline{r}}_{r l, r}^{r}
\end{array}\right]
$$

In order to calculate the normal and sliding velocity components, $\underline{\dot{r}}_{r l, r}^{r}=\left[\begin{array}{lll}v_{r x} & v_{r y} & v_{r z}\end{array}\right]^{T}$ needs to be transformed using the relative orientation angle $\varphi$ as follows:

$$
\left[\begin{array}{c}
v_{x-\text { slid }} \\
v_{\text {norm }} \\
v_{z-\text { slid }}
\end{array}\right]=\left[\begin{array}{ccc}
\cos \varphi & \sin \varphi & 0 \\
-\sin \varphi & \cos \varphi & 0 \\
0 & 0 & 1
\end{array}\right]\left[\begin{array}{l}
v_{r x} \\
v_{r y} \\
v_{r z}
\end{array}\right]
$$

The sliding velocity could be computed as:

$$
\underline{v}_{s}=\left|\underline{v}_{s}\right| \underline{u}_{v s} \quad \text { where } \quad\left|\underline{v}_{s}\right|=\sqrt{v_{x-s l i d}^{2}+v_{z-s l i d}^{2}} \text { and } \underline{u}_{v s}=\underline{v}_{s} /\left|\underline{v}_{s}\right|
$$

It should be noted that each section of the track rail will have different stiffness. If the roller is in contact with more than one link at the same time, a stiffness modification factor should be calculated based on the rail section profile. The normal contact force results from elastic spring force and damping force can be evaluated as follows:

$$
\begin{aligned}
& F_{n}=F_{\text {stiff }}+F_{\text {damp }}, \\
& \text { where } F_{\text {stiff }}=\kappa_{\text {sec }} K_{\text {nom }} \delta_{\text {norm }}^{1.5}, \\
& \quad F_{\text {damp }}=\kappa_{\text {sec }} C_{\text {nom }} v_{\text {norm }}
\end{aligned}
$$

where $K_{\text {nom }}$ is the rail nominal stiffness coefficient, $\kappa_{\text {sec }}$ correction factor to account for the change in the rail section thickness, $C_{\text {nom }}$ is the nominal damping coefficient; $\delta_{\text {norm }}$ and $v_{\text {norm }}$ are the penetration and normal component of the approach velocity. Similar to the sprocket contact module, the adverse effect of the large impact force on the friction force can be reduced by assuming the friction coefficient as smooth function of the sliding velocity as shown in Equation (35).

The contact and friction force vectors are rotated back to the roller reference frame as follows:

$$
\underline{F}^{c}=\left[\begin{array}{c}
F_{x} \\
F_{y} \\
F_{z}
\end{array}\right]=\left[\begin{array}{ccc}
\cos \varphi & -\sin \varphi & 0 \\
\sin \varphi & \cos \varphi & 0 \\
0 & 0 & 1
\end{array}\right]\left[\begin{array}{c}
F_{f x} \\
F_{n} \\
F_{f z}
\end{array}\right]
$$

Then contact forces are now translated to the sprocket reference frame and the link reference frame as follows:

$$
F_{r, r}^{r}=\left[\begin{array}{cc}
I & 0 \\
\tilde{\tilde{r}}_{r l}^{r} & I
\end{array}\right]^{-T}\left[\begin{array}{c}
0 \\
\underline{F}^{c}
\end{array}\right] \quad \text { and } \quad F_{l, l}^{l}=\left(X^{r l}\right)^{-T} F_{r, r}^{r}
$$

\section{Machine Terrain Interaction Link-Ground Contact}

Accurate modeling of the machine interaction with the environment is very crucial for accurate load prediction and machine response during the dynamic simulation. The dynamic response of the machine to environmental effects depends on several factors including (Bekker, 1965), (McCullough et al, 1985), (Rubinstein et al, 2004), (Sandu et al, 2005):

1. The magnitude, frequency, location, and direction of the dynamic load transmitted from soil to machine.

2. Geometry of the track link that interacts with soil.

3. The dynamic properties of the machine component.

4. The dynamic properties of the soil.

5. The terrain geometry and representation 
It is always desired that the machine-terrain interaction model be able to predict tractive performance as a function of vehicle design parameters, the soil parameters, and the operating conditions. Track terrain interaction typically includes calculating the following quantities: the under-track support pressure and force, the track-link sinkage, and the tractive shear force. Various models have been developed in the literature to predict the tractive performance of tracked vehicles. Those approaches include analytical models, empirical models, numerical models or some combination of them. Design engineers always need to achieve a fast yet accurate solution for the problems faced in earth moving equipment design. Advanced numerical models based on full finite element or mesh free methods are not the best option to achieve the designer goals because of the computational cost.

This paper presents an advanced semi-analytical and semi-numerical track soil model could be developed based on the fundamentals of soil mechanics and the theory of plasticity. Results of the proposed model obtained for tracked vehicles on different soil properties and irregular ground surfaces show that the model is capable of capturing the dynamics of the track-soil interaction. Although the track-soil model is computationally cheap, it has the following desired characteristics: nonlinear based model, provide adequate representation of the tractive force, compute the track penetration, account for soil resistance, provide turning capabilities, and can capture different soil types (cohesive and non-cohesive soils). The proposed model could be used with flat and irregular surfaces.

The regular and irregular ground surface can be defined based on the required course of simulations necessary. Flat surface could be sufficient for simulating excavation, dozing, or ride comfort where the machine could be driven in straight path. If the simulation requires driving the machine rough terrain, it will be necessary to describe the surface with more detailed geometry. In the proposed approach, the ground surface could be defined using triangular mesh where every ground patch is defined using three facets. The soil characteristics could be defined to be uniform over the ground surface or it may vary at different locations. If the soil characteristics are variable, the values of the dynamic properties could be defined at each facet. Once the contact point between the track shoe and the ground surface is determined, the soil characteristics can be interpolated from the values at the element facets. Assuming a triangular element with three facets $A\left(x_{1}, y_{1}, w_{1}\right), B\left(x_{2}, y_{2}, w_{2}\right), C\left(x_{3}, y_{3}, w_{3}\right)$ where $x$ and $y$ are the facet coordinates and $w$ is the dynamic property at the facet, as shown in Figure 12. It could be shown that the value of $w$ at the contact point, $D$, that is defined in the plane of the triangle is calculated as follows:

$$
w(x, y)=\frac{1}{2 A}\left\{\begin{array}{c}
{\left[\left(x_{2} y_{3}-x_{3} y_{2}\right)+\left(y_{2}-y_{3}\right) x+\left(x_{3}-x_{2}\right) y\right] w_{1}} \\
\quad+\left[\left(x_{3} y_{1}-x_{1} y_{3}\right)+\left(y_{3}-y_{1}\right) x+\left(x_{1}-x_{3}\right) y\right] w_{2} \\
\quad+\left[\left(x_{1} y_{2}-x_{2} y_{1}\right)+\left(y_{1}-y_{2}\right) x+\left(x_{2}-x_{1}\right) y\right] w_{3}
\end{array}\right\} \text { where } A=\frac{1}{2}\left|\begin{array}{lll}
1 & x_{1} & y_{1} \\
1 & x_{2} & y_{2} \\
1 & x_{3} & y_{3}
\end{array}\right|
$$

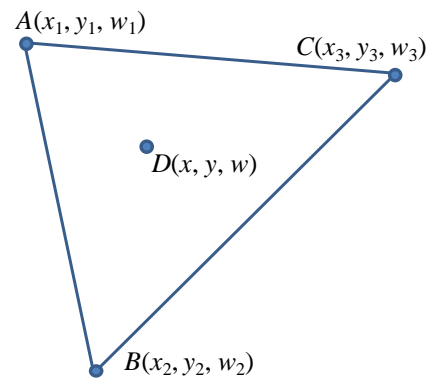

Figure 12. Representing the ground surface geometry using contact triangular element

It should be mentioned that in a preprocessing step, the parameter $A$, the normal and tangent vectors, $x_{t}$ and $y_{t}$, could be determined for each element and stored for later use during the dynamic simulation. From the surface normal and tangent vectors, an element rotational matrix, $R^{o e}$, relative to global could be determined and stored. These pre-computed quantities could save significant run-time during the simulation.

In the following analysis, the track shoe is approximated by a flat plate and the ground surface is described by a surface with its reference located at the inertial frame of reference. To improve the computational efficiency, the interaction algorithm identifies the track links in the proximity of the ground surface and saves them in a proximity list. For the following time steps, the algorithm checks the contact between the ground surface and the links in the list every time step. Since the track links move at slow rate, the list may not change for several time steps. If one of the links moves out of the proximity range, the detection algorithm reconstructs the proximity list. The proximity distance could be set as one track pitch. 
At every integration time step the interaction algorithm checks all the links in the proximity list and identifies whether the link is in contact with ground or not. During simulation, the track could be fully or partially penetrated in the ground and the interaction forces will vary over the track link depending on the stage of contact penetration. For this reason, the shoe plate could be divided into four shoe contact areas (SCA) where each SCA is identified by a shoe contact marker (SCM) at its center. The algorithm checks for the contact between the ground and the track links by determining the position and orientation between the link $l$ in global coordinate system $\underline{r}_{0 l}^{0}=\left[\begin{array}{lll}r_{x} & r_{y} & r_{z}\end{array}\right]^{T}$ and $\underline{R}^{0 l}$. The contact detection algorithm projects the origin of the SCM on the ground surface and determines which marker is in contact and identifies the ground contact triangular element (CTE). A contact point, $g$, is defined by the intersection between the $y$-axis of SCM and the plane of CTE. The relative position vector between the SCM $i$ and the contact point $g$ is determined as $r_{g l, i}$. The pre-determined surface element rotation matrix, $R^{0 e}$, is used to project $r_{g l, i}$ into the CTE coordinate system, as shown in Figure 13, and determine the penetration of that marker $\delta_{i}$. If $y_{g l, i}$ is less than the shoe plate height, then penetration occurred.

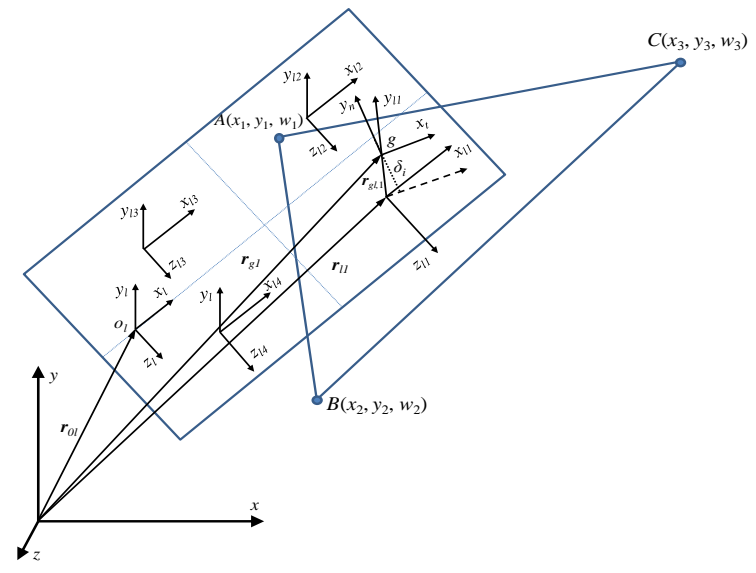

Figure 13. Link surface contact detection

The soil dynamic properties are interpolated at the contact point using Equation (47) and used to calculate the interaction forces. The pressure and the vertical force at marker $i$ are calculated using the sinkage as follows:

$$
p_{y, i}=K_{y} \delta_{i} \quad \text { and } \quad F_{y, i}=p_{y, i} A_{i}
$$

where $K_{y}$ is the vertical stiffness of the soil, $A_{i}$ is the area defined by the marker $i, p_{y, i}$ is the pressure at marker $i$ due to the penetration at that marker. The tractive force of the vehicle depends on the shear force between the track and soil in the longitudinal direction of the link. The shear stress could be calculated as follows:

$$
\tau=\left(K_{c}+p_{y, i} \tan \phi\right)
$$

where $K_{c}$ is the cohesion coefficient and $\varphi$ is the angle of internal friction. The relative position vector between the SCM and the contact point could be projected into the CTE coordinate system as follows:

$$
d_{g l}=\left(R^{0 e}\right)^{-1} \mathbf{r}_{g l}=\left[\begin{array}{lll}
d_{x} & d_{y} & d_{z}
\end{array}\right]^{T}
$$

The value of slip and slip directions could be determined from the kinematic link motion in the longitudinal and lateral directions in reference to Figure 13 as follows:

$$
s_{i}=\sqrt{d_{x}^{2}+d_{z}^{2}}, \quad u_{x i}=d_{x} / s_{i}, \quad \text { and } \quad u_{z i}=d_{z} / s_{i}
$$

The shear force acting at the area $A_{i}$ could be calculated as follows:

$$
F_{T}=\tau A_{i} \tanh \left(s_{i} k_{T i}\right)
$$

The lateral force and the tractive forces could be calculated on the ground as follows: 


$$
F_{T x, i}=F_{T} u_{x i}, \quad F_{T z, i}=F_{T} u_{z i}
$$

The damping forces depends on the direction link sinkage which could be determined by projecting the link velocity in CTE coordinate system as follows

$$
\dot{s}_{i}=\sqrt{v_{x i}^{2}+v_{z i}^{2}}, \quad \bar{u}_{x i}=v_{x i} / \dot{s}_{i}, \quad \bar{u}_{z i}=v_{z i} / \dot{s}_{i}
$$

The damping forces could be calculated based on the slip velocity in the $x-z$ plan as follows:

$$
F_{d i}=\dot{s}_{i} A_{i} C\left(1-e^{-\left|s_{i}\right|}\right) \text {, and } F_{d x, i}=F_{d} \bar{u}_{x i}, \quad F_{d z, i}=F_{d} \bar{u}_{z i}
$$

These forces are calculated in the CTE coordinate system and should be transformed back to the SCM. The forces at each marker could be calculated using the set of equations (48) - (55). The interaction forces and are accumulated at the link reference coordinate as follows:

$$
\left[\begin{array}{l}
F_{x} \\
F_{y} \\
F_{z}
\end{array}\right]=\left[\begin{array}{c}
\sum_{i=1}^{4} F_{T x, i}+F_{d x, i} \\
\sum_{i=1}^{4} F_{y, i} \\
\sum_{i=1}^{4} F_{T z, i}+F_{d z, i}
\end{array}\right]
$$

The moment vectors associated with these forces could be calculated at the link reference using cross product of the forces with the position vector of SCM.

\section{Proposed Algorithm for Solving the System Equations of Motion}

Figure 14 shows a schematic representation for a possible implementation of the proposed approach. The track super-component is modeled as an external module communicating with the general purpose multibody dynamics code. For this development, the general purpose multibody code is using the joint-based recursive formulation described in Section 2, (Featherstone, 2005), (Omar, 2014). Prior to solving the system equation of motion, all the bodies in the system must be assembled and the track-links must be wrapped around the sprockets, the carrier rollers, idlers and the track rollers without penetrating into those contacting bodies. The initial conditions of all bodies are determined to minimize the constraint violations and eliminate any interference between the metals especially contact with the track-links. Performing static equilibrium of the system before the simulation starts can improve the simulation stability. At the beginning of the dynamic simulation, the structure of the equation of motion is determined based on a preliminary analysis of the system topology. The dependent and independent variable sets are determined using the generalized coordinate partitioning approach. In this proposed algorithm, the solver integrates both dependent and independent variable sets and the kinematic constraints are enforced.

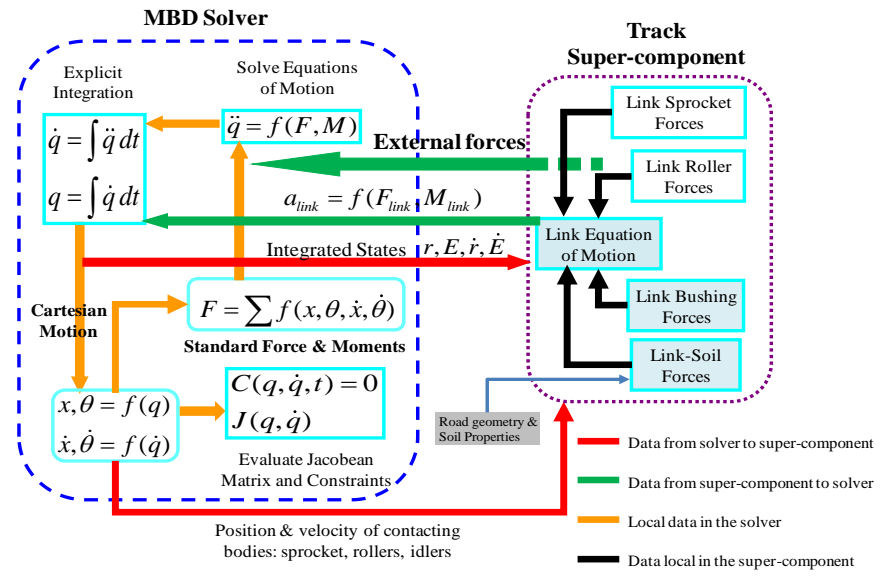

Figure 14. Interfacing the track super-component with multibody solver 
The input states sent to the integrator represent the first and second time derivative of the joint variables of the bodies in the main system evaluated using (6). Simultaneously, the equations of motion of the track-links are evaluated and the Cartesian accelerations are computed using (17). The vector of the track-links' states is appended to the main system states vector and sent to the integrator. The output states are the joint displacements and the joint velocities of all the bodies in the main system and the track-links. The following summarizes the structure and flow of the multibody dynamic simulation algorithm:

1. Using the joint variables returned from the integrator, the Cartesian displacements, velocities and accelerations are calculated. The information of the bodies in contact with the track links are sent to the track super-component for contact detection.

2. The Cartesian displacements and velocities of the track-links are calculated.

3. The internal and external forces acting on the bodies in the main system are calculated and applied to the different bodies using the standard approach.

4. The track super-components calculate the interaction forces, including:

4.1. Pin-bushing forces between the track links

4.2. Contact forces between the track-links and rollers. Perform re-boxing if needed.

4.3. Contact forces between links and sprockets. Perform re-boxing if needed.

4.4. Interaction forces between the track links and the ground. Update the proximity list if needed.

5. Apply the forces from the track links to the contacting bodies:

5.1. The contact forces between track-links and sprockets.

5.2. The contact forces between track-links and rollers and idlers.

6. Transform the inertia matrices of the bodies in the main system into global coordinate system.

7. For all the track-links, the inertia matrix, the centrifugal and Coriolis forces, and the external forces are transformed into the global local coordinate system.

8. Project the Cartesian forces into the body joint space.

9. Factor the mass matrix based on the independent and dependent variables sets.

10. Calculate second derivatives of the joint variables.

11. Calculate the inertia forces, centrifugal and Coriolis forces in Cartesian space.

12. Invert the track-links (floating body) mass matrix and calculate the body second derivatives.

13. Append the track- links states to the main system states.

14. Send the states to the integrator.

The proposed solver algorithm utilizes a predictor-corrector integrator with variable-order interpolation polynomials and variable time step (Bernan, Campbell, \& Petzold, 1996) and (Ascher \& Petzold, 1998). This explicit integrator insures the stability of the solution and the ability to capture the high-speed impacts between the different machine parts.

\section{Simulation Example}

The following example shows a full simulation of a tracked vehicle going in and out of V-ditch formed terrain. The kinematic tree of the tracked bulldozer is shown in Figure 15. The chassis of the machine is referenced to the ground and include most of the inertial components: structure, engine, tanks, etc. The cab is attached to the chassis with cab mounts. The left and right sprockets are connected to the chassis through the sprocket drive tandem (SDT). The equalizer bar (EB) is split into two bodies, left and right parts, where left EB part is connected to the chassis and the right EB is connect to right rear roller frame (RRF). Right and left pivoting shafts (PS) are attached to the chassis. The left and right rear roller frames are connected to the chassis through the pivoting shafts. Both left and right RRFs carry two carry rollers, and five track rollers and front roller frame (FRF). The idlers are connected to the front roller frames. The major standard force modules in the main system include the following:

- Flexibility of the pivoting shaft is modeled using elastic torsional spring element with structural damping.

- Torsional spring and damper is connecting the two sprocket tandems to the chassis to account for the bending stiffness of the drive tandem.

- Torsional springs and dampers are connecting the two parts of the equalizer bar.

- Recoil mechanisms are located between the front and rear roller frames are modeled using spring and dampers. 


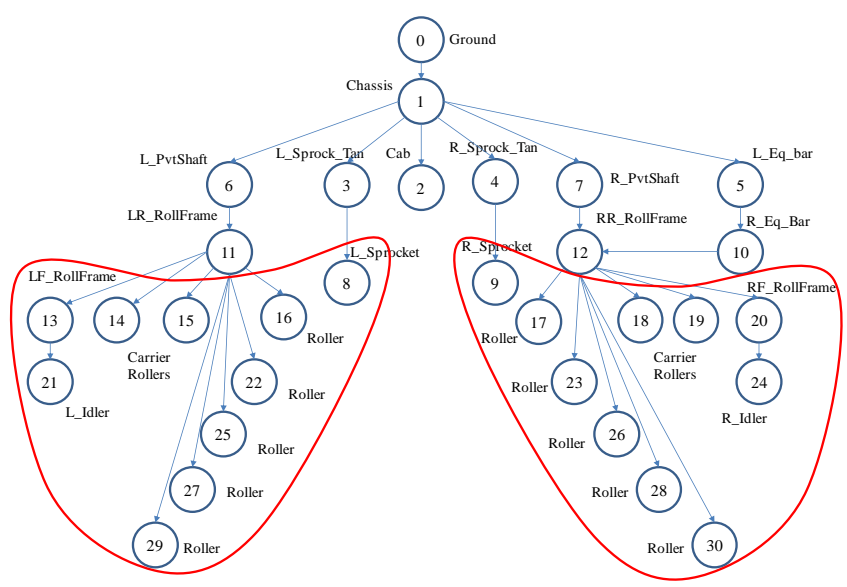

Figure 15. Kinematic tree of the tracked bulldozer.

Each side of the bulldozer track is modeled with a track super-component. The bodies in the main system that interact with the track-links are outlined in Figure 15. Each side of the track contains 46 track-links. The track-links are connected with bushing elements. The sprocket tooth profile used in this simulation is shown in Figure 6. The profile of the carrier rollers, track rollers, and idlers are shown in Figure 9. The profiles that describe rail geometry of the track-links at different sections are shown in Figure 10. Figure 16 shows a full simulation of a tracked vehicle going in and out of V-ditch formed terrain. As shown in the figure, the super-component is capable of handling most of the simulation requirements.

\section{Conclusions}

This paper presented a comprehensive approach for modeling tracked vehicles. The proposed approach represents a stand-alone super-component that can be integrated in any multibody dynamics code. The super-component interacts with the main code on the force level without affecting the structure of system equations. This super-component models the chain track links as individual bodies with six degrees of freedom each and their equations of motion are developed using spatial algebra. The connections between the links are modeled using a compliant bushing element that allows relative rotation and captures the elastic deformation of the pin-bushing connection. The links interact with the undercarriage bodies through two major force modules: link-roller interaction and link-sprocket interaction. The link-roller interaction is modeled using comprehensive geometry-based contact detection algorithm. Carrier roller, track rollers, and idlers are considered to be surface of revolutions and their surface could be described by simple profile.
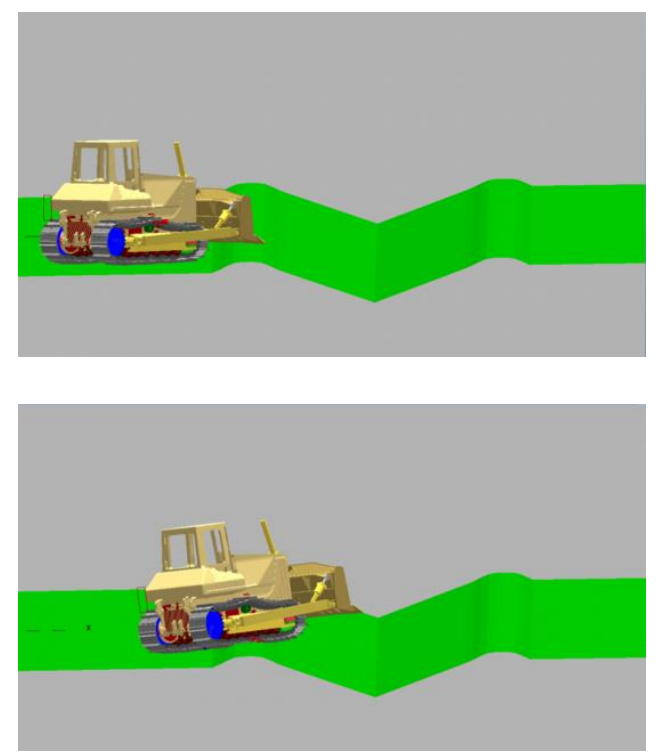

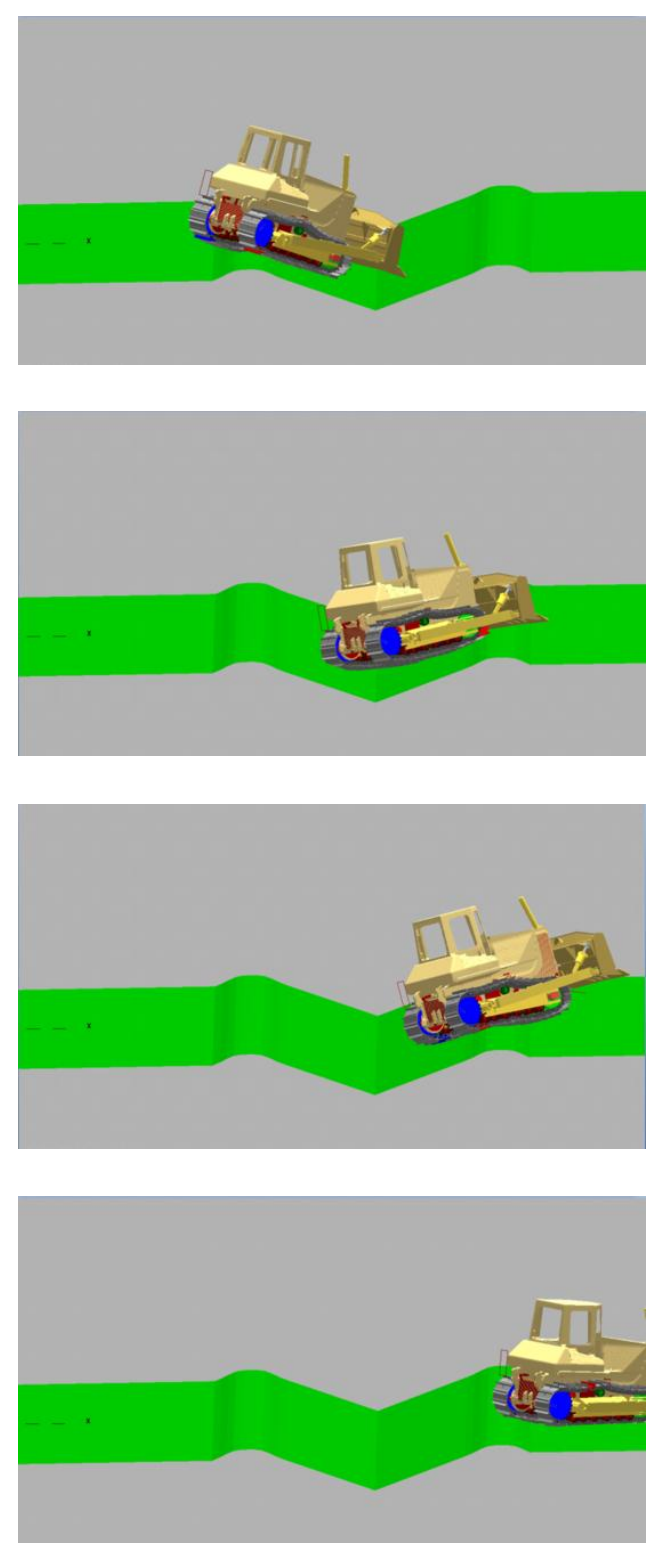

Figure 16. Tracked vehicle interacting V-ditch shaped ground surface

The force module benefits from this similarity to simplify the computational efforts. Another comprehensive force module used to model the interaction between the sprocket and track-links. The module allows the sprocket tooth to be described by a spline or by an approximate profile. One of the major developments in this paper is the track-terrain interaction module. A force module is developed to predict the contact point between the track shoe and the ground surface and determine the shoe penetration and sinkage. The module allows the irregular terrain geometry to be specified for comprehensive simulation requirements. The force module calculates the normal support force based on linear relation between the penetration and normal stresses. The tractive force is calculated based on nonlinear relation between slip and shear stress. The lateral forces acting on the link were calculated and projected on the shoe coordinate system. The paper presented the solution procedure and the required data exchange between the super-component and the main solver. To improve the computational efficiency of the super-component, preprocessing steps were proposed to eliminate the unnecessary repeated calculations during the run-time. The results of proposed modules were presented through a full vehicle simulation with terrain interaction. All the proposed force modules are independent of the main solver and could be replaced with more accurate or elaborate modules.

\section{References}

Ascher, U. M., \& Petzold, L. R. (1998). Computer Methods for Ordinary Differential Equations and Differential-Algebraic Equations. ISBN: 0898714125, Society for Industrial and Applied Mathematics, Philadelphia, PA. http://dx.doi.org/10.1137/1.9781611971392 
Bekker, M. G. (1969). Introduction to Terrain-Vehicle Systems., Ann Arbor, MI: University of Michigan Press.

Brenan, K. E., Campbell, S. L., \& Petzold, L.R. (1996). Numerical Solutions of Initial-Value Problems in Differential-Algebraic Equations, Siam, North-Holland, New York.

Choi, J. H., Lee H .C., \& Shabana, A. A. Spatial Dynamics of Multibody Tracked Vehicles Part II: Contact Forces and simulation Results. Vehicle System Dynamics, 29, 113-137. http://dx.doi.org/10.1080/00423119808969365

Choi, J. H., Lee H. C., \& Shabana, A. A. (1998). Spatial Dynamics of Multibody Tracked Vehicles Part I: Spatial Equations of Motion. Vehicle System Dynamics, 29, 27-49. http://dx.doi.org/10.1080/00423119808969365

Dubowsky, S. (1974). On Predicting the Dynamic Effects of Clearances in One-Dimensional Closed Loop Systems. Journal of Engineering for Industry, Series B, 96, 324-329. http://dx.doi.org/10.1115/1.3438321

Featherstone, R. (2005). Efficient Factorization of the joint Space Inertia Matrix for Branched Kinematic Trees. Int J of Robotics Research, 24(6), 487-500.

Featherstone, R. (2008). Rigid Body Dynamics Algorithms, New York, Springer. http://dx.doi.org/10.1177/0278364905054928

Flores, P., \& Ambrósio, J. (2010). On the Contact Detection for Contact- Impact Analysis in Multibody Systems. Multibody System Dynamics, 24(1), 103-122.

Flores, P., \& Ambrósio, J. (2004). Revolute Joints with Clearance in Multibody Systems. Computers \& Structures, 82, 1359-1369. http://dx.doi.org/10.1007/s11044-010-9209-8

Gottschalk, S., Lin, M.C., \& Manocha, D. (1996). OBB-Tree: A Hierarchical Structure for Rapid Interference Detection, University of North Carolina.

Janosi, Z., \& Hanamoto, B. (1961). An Analysis of the Drawbar Pull vs. Slip Relationship for Track Laying Vehicles. 1st ISTVS. Turin.

Johnson, K. L. (1994). Contact Mechanics, Cambridge University Press, Cambridge, United Kingdom.

Ma, Z. \& Perkins, N. C. (2006). A Super-Element of Track-Wheel-Terrain Interaction for Dynamic Simulation of Tracked Vehicles. Multibody System Dynamics, 15, 351-372. http://dx.doi.org/10.1007/s11044-005-9001-3

Madsen, J. (2007). High Fidelity Modeling and Simulation of Tracked Elements for Off-Road Applications Using MSC/ADAMS: Technical Report TR-2007-02, Simulation-Based Engineering Laboratory, University of Wisconsin, Madison.

Madsen, J., Pechdimaljian, N., \& Negrut, D. (2007). Penalty versus complementarity-based frictional contact of rigid bodies: a CPU time comparison: Technical Report TR-2007-05, Simulation-Based Engineering Laboratory, University of Wisconsin, Madison.

McCullough, M. K., \& Haug, E. J. (1985). Dynamics of High Mobility Track Vehicles. ASME Paper No. 85-DET-95.

MSC Software Corporation. (2007). ADAMS Standard Documentation and Help, MD/ADAMS R3.

Omar, M. A. (2014). Chain Drive Simulation Using Spatial Multibody Dynamics. Journal of Advances in Mechanical Engineering, Vol 2014, 378030.

Omar, M. A. (2014). Modeling Flexible Bodies in Multibody Systems in Joint Coordinates Formulation Using Spatial Algebra. Journal of Advances in Mechanical Engineering, Vol 2014, Article ID 468986. http://dx.doi.org/10.1155/2014/468986.

Omar, M. A. (2014). Static Analysis of Large-Scale Multibody System Using Joint Coordinates and Spatial Algebra Operator. The Scientific World Journal, Vol 2014, 409402.

Pedersen, S. L., Hansen, J. M., \& Ambrósio, J. A. (2004). Roller Chain Drive Model Including Contact with Guide-Bars. Multibody System Dynamics, 12, 285-301. http://dx.doi.org/10.1023/B:MUBO.0000049131.77305.d8

Pedersen, Sine Leergaard. (2004). Simulation and Analysis of Roller Chain Drive Systems, Phd dissertation, Technical University of Denmark.

Pereira, C., Ambrósio, J., \& Ramalho , A. (2011). Contact Mechanics In A Roller Chain Drive Using A Multibody Approach, 13th World Congress in Mechanism and Machine Science, Guanajuato, México, June.

Pereira, C., Ambrósio, J., Ramalho, A., \& Flores, P. (2010). A methodology for the generation of models for multibody chain drives. Multibody System Dynamics, 24(3), 303-324. http://dx.doi.org/10.1007/s11044-010-9207-x

Pereira, C., Ramalho, A., \& Ambrósio, J. A. Critical Overview of Internal and External Cylindrical Contact Force Models. Nonlinear Dynamics. http://dx.doi.org/10.1007/s11071-010-9830-3, 2010 
Petzold, L., \& Wehage, R. (1994). Real Time Simulation of Large Scal Multibody Systems Using Automated Equation Decoupling Techniques. US Army Research Office.

Roberson, R. E., \& Ming-Ming, Z. (1986). Generalized correction of numerical errors in kinematical differential equations based on Euler normalized parameters. Acta Mechanica, 59(3-4), 133-138. http://dx.doi.org/10.1007/BF01181660

Rubinstein, D., \& Hitron, R. (2004). A detailed multi-body model for dynamic simulation of offroad tracked vehicles. Journal of Terramechanics, 41, 163-173. http://dx.doi.org/10.1016/j.jterra.2004.02.004

Ryu, H. S., Bae, D. S., Choi, J. H., \& Shabana, A. A. (2000). A compliant track link model for high speed, high-mobility tracked vehicles. Intl Journal for Numerical Methods in Engineering, 48, 1481-1502. http://dx.doi.org/10.1002/1097-0207(20000810)48:10<1481::AID-NME959>3.0.CO;2-P.

Ryu, H. S., Huh, K. S., Bae, D.S., \& Choi, J.H. (2003). Development of a Multibody Dynamics Simulation Tool for Tracked Vehicles (Part I, Efficient Contact and Nonlinear Dynamics Modeling). JSME International Journal, 46(2).

Sandu, C. \& Freeman, J. S. (2005). Military tracked vehicle model. Part I: multibody dynamic formulation. International Journal of Vehicle Systems Modelling and Testing, 1(1/2/3), 48-67.

Shabana, A. A. (2001). Computational Dynamics, John Willy \& Sons, Inc., Second edition.

Shampine, L., \& Gordon, M. (1975). Computer Solution of Ordinary Differential Equations: The Initial Value Problem, Freeman, San Francisco, California.

Stronge, W. J. (2004). Impact Mechanics, Cambridge University Press.

Wong, J. Y. (1983). Evaluation of Soil Strength Measurements, Report no. NRCC 22881, Division of Energy, National Research Council of Canada.

Wong, J. Y., \& Bekker, M. G. Terrain Vehicle Systems Analysis Monograph, Department of Mechanical and Aerospace Engineering: Carleton University, Ottawa, Ont., Canada. 1976-78, 1980 and 1985. http://dx.doi.org/10.1016/0022-4898(83)90028-9

Wong, J. Y., Garber, M., \& Preston-Thomas, J. (1984). Theoretical Prediction and Experimental Substantiation of the Ground Pressure Distribution and Tractive Performance of Tracked Vehicles. Transport Engineering, 198(D15).

Wong, J. Y. (1989). Terramechanics and Off-Road Vehicles., Amsterdam: Elsevier Science.

Wong, J. Y., \& Preston-Thomas, J. (1983 ). On the Characterization of the Shear-Stress-Displacement Relationship of Terrain. Journal of Terramechanics, 19(4).

Wong, J. Y., (2001). Theory of Ground Vehicles. 3rd ed, New York: Wiley Interscience.

\section{(cc) BY}

This work is licensed under a Creative Commons Attribution 3.0 License. 